\title{
Linear Diophantine Uncertain Linguistic Power Einstein Aggregation Operators and Their Applications to Multiattribute Decision Making
}

\author{
Tahir Mahmood (D, ${ }^{1}$ Izatmand, ${ }^{1}$ Zeeshan Ali, ${ }^{1}$ Kifayat Ullah $\mathbb{D}^{2},{ }^{2}$ Qaisar Khan, ${ }^{3}$ \\ Ahmed Alsanad $\left(\mathbb{D},{ }^{4}\right.$ and Mogeeb A. A. Mosleh $\mathbb{1}^{5}$ \\ ${ }^{1}$ Department of Mathematics \& Statistics, International Islamic University Islamabad, Islamabad, Pakistan \\ ${ }^{2}$ Department of Mathematics, Riphah Institute of Computing and Applied Sciences, Riphah International University Lahore, \\ Lahore 54000, Pakistan \\ ${ }^{3}$ Department of Mathematics, University of Haripur, Haripur, Pakistan \\ ${ }^{4}$ STC's Artificial Intelligence Chair, Department of Information Systems, College of Computer and Information Sciences, \\ King Saud University, Riyadh 11451, Saudi Arabia \\ ${ }^{5}$ Faculty of Engineering and Information Technology, Taiz University, Taiz 6803, Yemen
}

Correspondence should be addressed to Ahmed Alsanad; aasanad@ksu.edu.sa and Mogeeb A. A. Mosleh; mogeebmosleh@taiz.edu.ye

Received 17 April 2021; Accepted 17 July 2021; Published 5 August 2021

Academic Editor: Abdel-Haleem Abdel-Aty

Copyright (c) 2021 Tahir Mahmood et al. This is an open access article distributed under the Creative Commons Attribution License, which permits unrestricted use, distribution, and reproduction in any medium, provided the original work is properly cited.

\begin{abstract}
Linear Diophantine uncertain linguistic set (LDULS) is a modified variety of the fuzzy set (FS) to manage problematic and inconsistent information in actual life troubles. LDULS covers the grade of truth, grade of falsity, and their reference parameters with the uncertain linguistic term (ULT) with a rule $0 \leq \alpha_{\mathrm{AMG}} u_{\mathrm{AMG}}(x)+\beta_{\mathrm{ANG}} v_{\mathrm{AMG}}(x) \leq 1$, where $0 \leq \alpha_{\mathrm{AMG}}+\beta_{\mathrm{ANG}} \leq 1$. In this study, the principle of LDULS and their useful laws are elaborated. Additionally, the power Einstein (PE) aggregation operator $(\mathrm{AO})$ is a conventional sort of $\mathrm{AO}$ utilized in innovative decision-making troubles, which is effective to aggregate the family of numerical elements. To determine the interrelationship between any numbers of arguments, we elaborate the linear Diophantine uncertain linguistic PE averaging (LDULPEA), linear Diophantine uncertain linguistic PE weighted averaging (LDULPEWA), linear Diophantine uncertain linguistic PE geometric (LDULPEG), and linear Diophantine uncertain linguistic PE weighted geometric (LDULPEWG) operators; then, we discuss their useful results. Conclusively, a decision-making methodology is utilized for the multiattribute decision-making (MADM) dilemma with elaborated information. A sensible illustration is specified to demonstrate the accessibility and rewards of the intended technique by comparison with certain prevailing techniques. The intended AOs are additional comprehensive than the prevailing ones to exploit the ambiguous and inaccurate knowledge. Numerous remaining operators are chosen as individual incidents of the suggested one. Ultimately, the supremacy and advantages of the elaborated operators are also discussed with the help of the geometrical form to show the validity and consistency of explored operators.
\end{abstract}

\section{Introduction}

MADM is a technique to discover the ideal option between a family of possibilities and a family of certain opinions. The perception of MADM has extensively been utilized in numerous disciplines. Ambiguity usually happened in genuine life troubles owing to the contribution of some convoluted restrictions, unavailability of evidence/information, and unpredictability of the challenge. To survive with this worry, Zadeh [1] expounded the theory of the fuzzy set (FS), which covers the grade of truth $u_{\mathrm{AMG}}(x)$ restricted to $[0,1]$. When the theory of FS was elaborated, then numerous intellectuals 
have exploited it in the natural environment of several areas. For instance, Molodtsov [2] initiated the soft sets, Mahmood [2] elaborated the new bipolar soft sets, Somjanta et al. [3] utilized the fuzzy sets in UP-algebra, Noppharat Dokkhamdang et al. [4] utilized the generalized fuzzy sets in UPalgebra, Tanamoon et al. [5] explored the Q-fuzzy sets and utilized in UP-algebra, Kawila et al. [6] explored the bipolar fuzzy UP-algebra, Mahmood and Hayat [7] initiated the characterizations of hemirings by their bipolar-valued fuzzy h-ideals, and Mahmood et al. [8] initiated the lattice ordered soft near ring. However, in certain situations, the principle of FS cannot be working effectively; for instance, when an individual face such sorts of information, which contains the truth and falsity grades, then FS failed. To survive with such circumstances, Atanassov [9] elaborated the theory of intuitionistic FS (IFS), which covers the grade of truth and falsity grades with the rule $0 \leq u_{\mathrm{AMG}}(x)+v_{\mathrm{ANG}}(x) \leq 1$. When the theory of IFS was elaborated, then numerous scholars have utilized it in the environment of several areas. For instance, Beg and Rashid [10] initiated the intuitionistic hesitant fuzzy sets, Atanassov [11] explored the intervalvalued intuitionistic fuzzy sets, Kumari and Mishra [12] investigated the parametric measures for IFSs, Jana and Pal [13] explored bipolar intuitionistic fuzzy soft sets, Joshi and Kumar [14] developed the fuzzy time series model based on intuitionistic fuzzy sets, Fu et al. [15] proposed the correlation measures by using the interval-valued intuitionistic fuzzy sets, and Meng and He [16] elaborated the geometric interaction aggregation operators by using the intuitionistic fuzzy sets.

Again, in certain situations, the principle of IFS cannot be working effectively; for instance, when an individual faces such sorts of information which contains the truth and falsity grades with the rule $0 \leq u_{\mathrm{AMG}}^{2}(x)+v_{\mathrm{ANG}}^{2}(x) \leq 1$, then IFS failed. To survive with such circumstances, Yager [17] elaborated the theory of Pythagorean FS (PFS), which covers the grade of truth and falsity grades with the rule $0 \leq u_{\mathrm{AMG}}^{2}(x)+v_{\mathrm{ANG}}^{2}(x) \leq 1$. When the theory of PFS was elaborated, then numerous scholars have utilized it in the environment of several areas. For instance, Garg [18] explored the linguistic PFS, Wei and Wei [19] developed the similarity measures for PFS, Xiao and Ding [20] investigated the diverges measures by using the PFS and their application in decision making, Ullah et al. [21] explored some similarity measures based on a novel complex PFS and their application in pattern recognition, $\mathrm{Li}$ and $\mathrm{Lu}$ [22] proposed some similarity and distance measures for PFSs, Garg [23] proposed the novel improved accuracy function for intervalvalued PFS and their application in decision making, and Yang and Hussain [24] investigated some entropy measures for PFS and their application.

Again, in certain situations, the principle of PFS cannot be working effectively; for instance, when an individual face such sorts of information which contains the truth and falsity grades with the rule $0 \leq u_{\mathrm{AMG}}^{q}(x)+v_{\mathrm{ANG}}^{q}(x) \leq 1$, then PFS failed. To survive with such circumstances, Yager [25] elaborated the theory of q-rung orthopair FS (q-ROFS), which covers the grade of truth and falsity grades with the rule $0 \leq u_{\mathrm{AMG}}^{q}(x)+v_{\mathrm{ANG}}^{q}(x) \leq 1$. When the theory of
q-ROFS was elaborated, then numerous scholars have utilized it in the environment of several areas; for instance, Ali [26] created another view on q-ROFSs. Liu and Wang [27] elaborated the aggregation operators for q-ROFSs. Peng and Liu [28] investigated the information measures for q-ROFSs. Wang et al. [29] explored the similarity measures for q-ROFSs. Ali and Mahmood [30] investigated the complex q-ROFSs and their Maclaurin symmetric mean operators. Liu et al. [31] proposed cosine similarity and distance measures for q-ROFSs. Liu and Wang [32] investigated the Archimedean Bonferroni mean operators for q-ROFSs. Liu et al. [33] explored the linguistic q-ROFSs. Garg [34] investigated the possibility degree for interval-valued q-ROFSs. The power aggregation operators for complex q-ROFSs were developed by Garg et al. [35].

To handle such sorts of concerns, Zadeh [36] investigated the theory of linguistic variable (LV) to describe the preferences of decision-makers. Moreover, the theory of a 2-tuple linguistic set was developed by Herrera and Martinez [37]. Liu and Jin [38] investigated the uncertain LV (ULV). In approximate actual existence troubles, the sum of truth and falsity grades to which an option filling an ascribe offered by the decisionmaker could not hold the rule of IFS, PFS, and q-ROFSs; then, the theory of IFS and PFS fails in such situations. To survive with such circumstances, Riaz and Hashmi [39] elaborated the theory of linear Diophantine FS (LDFS), which covers the grade of truth and falsity grades and their reference parameters with the rule $0 \leq \alpha_{\mathrm{AMG}} u_{\mathrm{AMG}}(\varrho)+\beta_{\mathrm{ANG}} v_{\mathrm{AMG}}(\varrho) \leq 1$. When the theory of LDFS was elaborated, then numerous scholars have utilized it in the environment of several areas; for instance, Riaz et al. [40] discovered the theory of linear Diophantine fuzz soft rough sets and their applications. Some algebraic structures based on LDFs were developed by Kamaci [41]. But, up to date, no study explored the theory of LDULVs and their operational laws.

The concepts of intuitionistic fuzzy sets (IFSs), Pythagorean fuzzy sets (PFSs), q-rung orthopair fuzzy sets (qROFSs), and linear Diophantine fuzzy sets have numerous applications in various fields of real life, but these theories have their own limitations related to the membership and nonmembership grades. To eradicate these restrictions, we introduce the novel concept of the linear Diophantine uncertain linguistic set (LDULS) with the addition of reference parameters and uncertain linguistic terms. The proposed model of LDULS is more efficient and flexible rather than other approaches due to the use of reference parameters and ULVs. LDULS also categorizes the data in MADM problems by changing the physical sense of reference parameters and ULVs. This set covers the spaces of existing structures and enlarge the space for membership and nonmembership grades with the help of reference parameters and ULVs. The motivation of the proposed model is given step by step in the whole manuscript. Now, we discuss some important objectives of this study.

(1) The theory of LDULS is more generalized than IFSs, PFSs, q-ROFSs, LDFSs, and ULVs

(2) If we choose the information in the form of $(0.5,0.6)$, then by using the condition of IFSs, that is, the sum 
of both terms is limited to unit interval, but $0.5+0.6=1.1>1$, the theory of IFS has been failed for coping with such sorts of issues, and the theory of LDULS is very comfortable to resolve the above issues. For this, we choose the reference parameters such as $(0.1,0.2)$; then, by using the condition of LDULS, $0.1 * 0.5+0.2 * 0.6=0.05+0.12=0.17<1$. We clear that the IFS is the special case of the proposed LDULS.

(3) If we choose the information in the form of $(0.8,0.9)$, then by using the condition of PFSs, that is, the sum of the square of both terms is limited to unit interval, but $0.8^{2}+0.9^{2}=0.64+0.81=1.45>1$, the theory of PFS has been failed for coping with such sorts of issues, and the theory of LDULS is very comfortable to resolve the above issues. For this, we choose the reference parameters such as $(0.2,0.2)$; then, by using the condition of LDULS, $0.2 * 0.8+0.2$ $* 0.9=0.16+0.18=0.34<1$. We clear that the theory of PFS is the special case of the proposed LDULS.

(4) If we choose the information in the form of $(0.1,0.1)$, then by using the condition of q-ROFSs, that is, the sum of the $q$-powers of both terms is limited to unit interval, but $1+1=2>1$, the theory of q-ROFS has been failed for coping with such sorts of issues, and the theory of LDULS is very comfortable to resolve the above issues. For this, we choose the reference parameters such as $(0,0.1)$; then, by using the condition of LDULS, $0.0 * 1+$ $0.1 * 1=0+0.1=0.1<1$. We clear that the theory of q-ROFS is the special case of the proposed LDULS.

(5) If we choose the information in the form of $\left(\left[s_{1}, s_{2}\right],(0.5,0.3),(0.5,0.4)\right)$, then using the condition of IFSs, PFSs, q-ROFSs, and LDFS has been failed; for coping with such sorts of issues, the theory of LDULS is very proficient and reliable technique to resolve with it. From the above analysis, the theories of IFSs, PFSs, q-ROFSs, and LDFSs are the special case of the proposed LDULS.

The opinions of IFSs, PFSs, q-ROFSs, and LDFSs have repeated applications in uncountable fields of genuine existence, but these philosophies have their shortcomings associated with the truth and falsity grades. To eliminate these constraints, we announce the narrative hypothesis of LDULS with the supplement of situation parameters. The suggested version of LDULS is additionally inexpensive and accommodating more accurately than other methodologies expected to the usage of suggestion parameters. LDULS also compartmentalizes the information in MADM troubles by modifying the physical meaning of orientation parameters. This set encompasses the areas of accessible assemblies and expands the space for truth and falsity grades with the help of reference parameters. The inspiration of the suggested pattern is offered step by step in the entire script.
The rest of this manuscript as follows. In Section 2, we briefly recall some definitions such as LDFSs, ULSs, and their operational laws. The theory of power aggregation (PA) operator is also reviewed. In Section 3, we notified the novel idea of LDULS and elaborated their fundamental laws. In Section 4, we elaborated the LDULPEA, LDULPEWA, LDULPEG, and LDULPEWG operators and their properties are also discovered. In Section 5, by using these operators, we acquire a MADM procedure based on elaborated operators. To determine the consistency and validity of the elaborated operators, we illustrate some examples by using explored operators. Finally, the superiority and comparative analysis of the elaborated operators with some existing operators are also determined and justify with the help of a graphical point of view. In Section 6, we discussed the conclusion of this study.

As shown above, the advantages of the operators and keeping the superiority of the elaborated approaches, the main points of the elaborated approaches are discussed as shown in Figure 1.

\section{Preliminaries}

Certain scholars have proposed numerous theories such as fuzzy sets, intuitionistic fuzzy sets, and their extensions to cope with awkward and complicated information in real-life issues. But these theories have their own limitations related to the membership and nonmembership grades. To eradicate these restrictions, the theory of the linear Diophantine fuzzy set (LDFS) with the addition of reference parameters was explored by Riaz and Hashmi [39]. The proposed model of LDFS is more efficient and flexible rather than other approaches due to the use of reference parameters. LDFS also categorize the data in MADM problems by changing the physical sense of reference parameters. This set covers the spaces of existing structures and enlarge the space for membership and nonmembership grades with the help of reference parameters. Keeping the advantages of the existing LDFS, in this section, we briefly recall some definitions such as LDFSs, ULSs, and their operational laws. The theory of the PA operator is also reviewed. In an overall study, the universal set is denoted by $X$.

Definition 1 (see [39]). A LDFS $A_{\mathrm{LD}}$ is elaborated by

$$
A_{\mathrm{LD}}=\left\{\frac{\left(x,\left(u_{\mathrm{AMG}}(x), v_{\mathrm{ANG}}(x)\right),\left(\alpha_{\mathrm{AMG}}, \beta_{\mathrm{ANG}}\right)\right)}{x \in X}\right\},
$$

with a rule $0 \leq \alpha_{\mathrm{AMG}} u_{\mathrm{AMG}}(x)+\beta_{\mathrm{ANG}} v_{\mathrm{AMG}}(x) \leq 1$, with $0 \leq \alpha_{\mathrm{AMG}}+\beta_{\mathrm{ANG}} \leq 1$. The symbol $\xi_{A_{\mathrm{LD}}} \pi(x)_{A_{\mathrm{LD}}}=1-\left(\alpha_{\mathrm{AMG}}\right.$ $\left.u_{\mathrm{AMG}}(x)+\beta_{\mathrm{ANG}} v_{\mathrm{ANG}}(x)\right)$ expressed the refusal grade. Simply, $A_{\mathrm{LD}}=\left(\left(u_{\mathrm{AMG}}(x), t v_{\mathrm{ANG}} n(x)\right), t\left(\alpha_{\mathrm{AMG}}, \beta_{\mathrm{ANG}}\right)\right)$ is called linear Diophantine fuzzy number (LDFN). To determine the interrelationships between any numbers of attributes, we revised some algebraic operation laws which are very important for proposed work in the next study. 


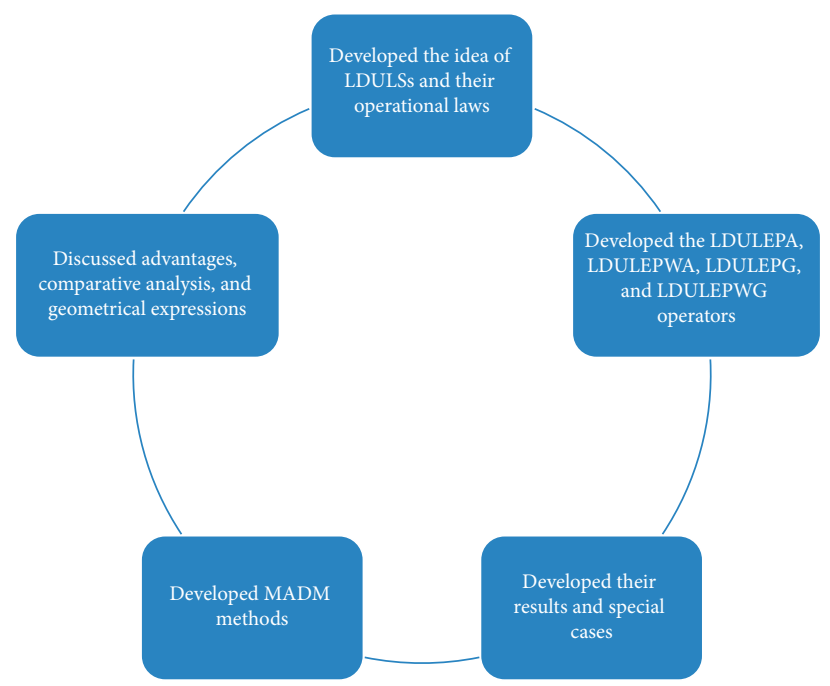

Figure 1: Geometrical expressions of the explored approaches.

Definition 2 (see [39]). For any two LDFNs $A_{\mathrm{LD}}=\left(\left(u_{\mathrm{AMG}}\right.\right.$ $\left.\left.(x), t v_{\mathrm{ANG}} n(x)\right), t\left(\alpha_{\mathrm{AMG}}, \beta_{\mathrm{ANG}}\right)\right)$ and $B_{\mathrm{LD}}=\left(\left(u_{\mathrm{BMG}}(x), t\right.\right.$ $\left.\left.v_{\mathrm{BNG}} n(x)\right), t\left(\alpha_{\mathrm{BMG}}, \beta_{\mathrm{BNG}}\right)\right)$,

$$
\begin{aligned}
A_{\mathrm{LD}} \oplus B_{\mathrm{LD}} & =\left(\left(u_{\mathrm{AMG}}+u_{\mathrm{BMG}}-u_{\mathrm{AMG}} u_{\mathrm{BMG}}, v_{\mathrm{AMG}} v_{\mathrm{BMG}}\right),\left(\alpha_{\mathrm{AMG}}+\alpha_{\mathrm{BMG}}-\alpha_{\mathrm{AMG}} \alpha_{\mathrm{BMG}}, \beta_{\mathrm{ANG}} \beta_{\mathrm{BNG}}\right)\right), \\
A_{\mathrm{LD}} \otimes B_{\mathrm{LD}} & =\left(\left(u_{\mathrm{AMG}} u_{\mathrm{BMG}}, v_{\mathrm{AMG}}+v_{\mathrm{BMG}}-v_{\mathrm{AMG}} v_{\mathrm{BMG}}\right),\left(\alpha_{\mathrm{AMG}} \alpha_{\mathrm{BMG}}, \beta_{\mathrm{ANG}}+\beta_{\mathrm{BNG}}-\beta_{\mathrm{ANG}} \beta_{\mathrm{BNG}}\right)\right), \\
\lambda A_{\mathrm{LD}} & =\left(\left(\left(1-\left(1-u_{\mathrm{AMG}}(x)\right)^{\lambda},\left(v_{\mathrm{ANG}}(x)\right)^{\lambda}\right),\left(1-\left(1-\alpha_{\mathrm{AMG}}\right)^{\lambda},\left(\beta_{\mathrm{ANG}}\right)\right)^{\lambda}\right)\right), \\
A_{\mathrm{LD}}^{\lambda} & =\left(\left(\left(u_{\mathrm{AMG}}(x)\right)^{\lambda}, 1-\left(1-\left(v_{\mathrm{ANG}}(x)\right)^{\lambda}\right)\right),\left(\left(\alpha_{\mathrm{AMG}}\right)^{\lambda}, 1-\left(1-\beta_{\mathrm{ANG}}\right)^{\lambda}\right)\right) .
\end{aligned}
$$

Furthermore, by using any number of LDFNs, we revised the score values for determining the ordering between any two LDFNs.

Definition 3 (see [39]). For any $\mathrm{LDFN} A_{\mathrm{LD}_{1}}=\left(\left(u_{\mathrm{AMG}_{1}}\right.\right.$ $\left.\left.(x), t v_{\mathrm{ANG}_{1}} n(x)\right), t\left(\alpha_{\mathrm{AMG}_{1}}, \beta_{\mathrm{ANG}_{1}}\right)\right)$, the score value $(\mathrm{SV})$ is elaborated by

$$
\varsigma_{A_{\mathrm{LD}}}=\varsigma\left(A_{\mathrm{LD}}\right)=\frac{1}{2}\left(u_{\mathrm{AMG}}(x)-v_{\mathrm{ANG}}(x)+(\alpha+\beta)\right) .
$$

Furthermore, if equation (6) has been failed by comparing any number of LDFNs, then we used the technique of accuracy function, so, for this, we revised the accuracy values for determining the ordering between any two LDFNs.

Definition 4 (see [39]). For any $\mathrm{LDFN} A_{\mathrm{LD}_{1}}=\left(\left(u_{\mathrm{AMG}_{1}}\right.\right.$ $\left.\left.(x), t v_{\mathrm{ANG}_{1}} n(x)\right), t\left(\alpha_{\mathrm{AMG}_{1}}, \beta_{\mathrm{ANG}_{1}}\right)\right)$, the accuracy value (AV) is elaborated by

$$
\varrho_{A_{\mathrm{LD}}}=\varrho\left(A_{\mathrm{LD}}\right)=\frac{1}{2}\left(\frac{\left(u_{\mathrm{AMG}}(x)+v_{\mathrm{ANG}}(x)\right)}{2}+(\alpha+\beta)\right) \text {. }
$$

Definition 5 (see [39]). For any two LDFNs $A_{\mathrm{LD}}=\left(\left(u_{\mathrm{AMG}}(x), t v_{\mathrm{ANG}} n(x)\right), t\left(\alpha_{\mathrm{AMG}}, \beta_{\mathrm{ANG}}\right)\right) \quad$ and $B_{\mathrm{LD}}=\left(\left(u_{\mathrm{BMG}}(x), t v_{\mathrm{BNG}} n(x)\right), t\left(\alpha_{\mathrm{BMG}}, \beta_{\mathrm{BNG}}\right)\right)$,

(1) If $\varsigma_{A_{\mathrm{LD}}}<\varsigma_{B_{\mathrm{LD}}}$, then $A_{\mathrm{LD}}<B_{\mathrm{LD}}$

(2) If $\varsigma_{A_{\mathrm{LD}}}>\varsigma_{B_{\mathrm{LD}}}$, then $A_{\mathrm{LD}}>B_{\mathrm{LD}}$

(3) If $\varsigma_{A_{\mathrm{LD}}}=\varsigma_{B_{\mathrm{LD}}}$, then

(1) If $\varrho_{A_{\mathrm{LD}}}<\varrho_{B_{\mathrm{LD}}}$, then $A_{\mathrm{LD}}<B_{\mathrm{LD}}$

(2) If $\varrho_{A_{\mathrm{LD}}}>\varrho_{B_{\mathrm{LD}}}$, then $A_{\mathrm{LD}}>b_{\mathrm{LD}}$

(3) If $\varrho_{A_{\mathrm{LD}}}=\varrho_{B_{\mathrm{LD}}}$, then $A_{\mathrm{LD}} \approx B_{\mathrm{LD}}$

When faced with problems that are too complex or illdefined to be solved by quantitative expressions, linguistic variables can be an effective tool because the use of linguistic information enhances the reliability and flexibility of classical decision models. To handle such sorts of concerns, Zadeh [36] investigated the theory of linguistic variable (LV) to describe the preferences of decision-makers. Moreover, the theory of a 2-tuple linguistic set was developed by Herrera and Martinez [37]. Liu and Jin [38] investigated the uncertain LV (ULV).

Definition 6 (see [36]). A LTS is elaborated by 


$$
S=\left\{s_{1}, s_{2}, \ldots, s_{l-1}\right\}
$$

where $l$ should be odd and hold the following conditions:

(1) If $l>l^{\prime}$, then $s_{l}>s_{l^{\prime}}$

(2) The negative operator neg $\left(s_{l}\right)=s_{l^{\prime}}$ with a condition $l+l^{\prime}=l+1$

(3) If $l \geq l^{\prime}, \max \left(s_{l}, s_{l^{\prime}}\right)=s_{l}$, and if $l \leq l^{\prime}, \max \left(s_{l}, s_{l^{\prime}}\right)=s_{l}$.

Let $\widetilde{s}=\left[s_{a}, s_{b}\right]$ where $s_{a}, s_{b} \in S, s_{a}$ and $s_{b}$ are the lower and the upper limits, respectively. We call $\widetilde{s}$ the uncertain linguistic variable. To determine the interrelationships between any numbers of attributes, we revised some operation laws for ULVs which are very important for the proposed work in next study.

Definition 7 (see [38]). For any two ULVs $\widetilde{s}_{1}=\left[s_{a 1}, s_{b 1}\right]$ and $\widetilde{s}_{2}=\left[s_{a 2}, s_{b 2}\right]$,

$$
\begin{aligned}
\widetilde{s}_{1} \oplus \widetilde{s}_{2} & =\left[s_{a 1}, s_{b 1}\right] \oplus\left[s_{a 2}, s_{b 2}\right]=\left[s_{a 1+a 2}, s_{b 1+b 2}\right], \\
\widetilde{s}_{1} \otimes \widetilde{s}_{2} & =\left[s_{a 1}, s_{b 1}\right] \otimes\left[s_{a 2}, s_{b 2}\right]=\left[s_{a 1 \times a 2}, s_{b 1 \times b 2}\right], \\
\lambda \widetilde{s}_{1} & =\lambda\left[s_{a 1}, s_{b 1}\right]=\left[s_{\lambda * a 1}, s_{\lambda * b 1}\right], \\
\left(\widetilde{s}_{1}\right)^{\lambda} & =\left[s_{(a 1)}, s_{(b 1)^{\lambda}}\right] .
\end{aligned}
$$

Definition 8 (see [33]). The PA operator is elaborated by

$$
\operatorname{PA}\left(A_{\mathrm{LD}-1}, A_{\mathrm{LD}-2}, \ldots, A_{\mathrm{LD}-n}\right)=\frac{\sum_{i=1}^{n}\left(1+T\left(A_{\mathrm{LD}-i}\right)\right) * A_{\mathrm{LD}-i}}{\sum_{i=1}^{n}\left(1+T\left(A_{\mathrm{LD}-i}\right)\right)},
$$

where $T\left(A_{\mathrm{LD}-i}\right)=\sum_{j=1}^{n} \sup \left(A_{\mathrm{LD}-i}, A_{\mathrm{LD}-j}\right)$, and $\sup \left(A_{\mathrm{LD}-i}\right.$, $\left.A_{\mathrm{LD}-j}\right)$ means the support for $A_{\mathrm{LD}-i}$ from $A_{\mathrm{LD}-j}$, which holds the following rules:

$$
\begin{aligned}
& \text { (1) } \sup \left(A_{\mathrm{LD}-i}, A_{\mathrm{LD}-j}\right)=\sup \left(A_{\mathrm{LD}-j}, A_{\mathrm{LD}-i}\right) \\
& \text { (2) } \sup \left(A_{\mathrm{LD}-i}, A_{\mathrm{LD}-j}\right) \in[0,1] \\
& \text { (3) } \sup \left(A_{\mathrm{LD}-i}, A_{\mathrm{LD}-j}\right) \geq \sup \left(A_{\mathrm{LD}-k}, A_{\mathrm{LD}-l}\right), \text { if } \mid A_{\mathrm{LD}-i} \text {, } \\
& A_{\mathrm{LD}-j}|\leq| A_{\mathrm{LD}-k}, A_{\mathrm{LD}-l} \mid
\end{aligned}
$$

\section{Linear Diophantine Uncertain Linguistic Set}

As shown above, the existing theories are very useful in the circumstances of investigation of any theories. In this study, by using the existing theories, we elaborated on the novel concept of LDULSs and their operational laws. These investigated theories are also justifying with the help of examples.

Definition 9. A LDULS $A_{\mathrm{LDULS}}$ is elaborated by

$$
A_{\mathrm{LDULS}}=\left\{\frac{x\left[\left[s_{\theta(X)}, s_{\tau(X)}\right],\left(u_{\mathrm{AMG}}(x), v_{\mathrm{ANG}}(x)\right),\left(\alpha_{\mathrm{AMG}}, \beta_{\mathrm{ANG}}\right)\right]}{x \in X}\right\},
$$

with the rule $0 \leq \alpha_{\mathrm{AMG}} u_{\mathrm{AMG}}(x)+\beta_{\mathrm{ANG}} v_{\mathrm{AMG}}(x) \leq 1$, with $0 \leq \alpha_{\mathrm{AMG}}+\beta_{\mathrm{ANG}} \leq 1$. The symbol $\xi_{A_{\mathrm{LD}}} \pi(x)_{A_{\mathrm{LP}}}=1-$ $\left(\alpha_{\mathrm{AMG}} u_{\mathrm{AMG}}(x)+\beta_{\mathrm{ANG}} v_{\mathrm{ANG}}(x)\right)$ expressed the refusal grade with $\quad s_{\theta(X)}, s_{\tau(X)} \in \widetilde{S}$. Simply, $\quad A_{\mathrm{LD}}=\left(\left[s_{\theta(X)}, s_{\tau(X)}\right]\right.$, $\left.\left(u_{\mathrm{AMG}}(x), v_{\mathrm{ANG}}(x)\right),\left(\alpha_{\mathrm{AMG}}, \beta_{\mathrm{ANG}}\right)\right)$ expresses the linear Diophantine uncertain linguistic number (LDULN).
Definition 10. For any two LDULNs $A_{\mathrm{LD}}=\left(\left[s_{A-\theta(X)}\right)\right.$, $\left.\left.s_{A-\tau(X)}\right],\left(u_{\mathrm{AMG}}(x), v_{\mathrm{ANG}}(x)\right),\left(\alpha_{\mathrm{AMG}}, \beta_{\mathrm{ANG}}\right)\right)$ and $B_{\mathrm{LD}}=$

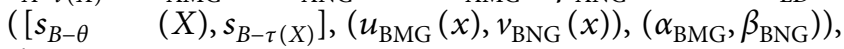
then

$$
\begin{aligned}
A_{\mathrm{LD}} \oplus B_{\mathrm{LD}} & =\left(\left[s_{A-\theta(X)+B-\theta(X)}, s_{A-\tau(X)+B-\tau(X)}\right],\left(u_{\mathrm{AMG}}+u_{\mathrm{BMG}}-u_{\mathrm{AMG}} u_{\mathrm{BMG}}, v_{\mathrm{AMG}} v_{\mathrm{BMG}}\right),\left(\alpha_{\mathrm{AMG}}+\alpha_{\mathrm{BMG}}-\alpha_{\mathrm{AMG}} \alpha_{\mathrm{BMG}}, \beta_{\mathrm{ANG}} \beta_{\mathrm{BNG}}\right)\right), \\
A_{\mathrm{LD}} \otimes B_{\mathrm{LD}} & =\left(\left[s_{A-\theta(X) * B-\theta(X)}, s_{A-\tau(X) * B-\tau(X)}\right],\left(u_{\mathrm{AMG}} u_{\mathrm{BMG}}, v_{\mathrm{AMG}}+v_{\mathrm{BMG}}-v_{\mathrm{AMG}} v_{\mathrm{BMG}}\right),\left(\alpha_{\mathrm{AMG}} \alpha_{\mathrm{BMG}}, \beta_{\mathrm{ANG}}+\beta_{\mathrm{BNG}}-\beta_{\mathrm{ANG}} \beta_{\mathrm{BNG}}\right)\right), \\
\lambda A_{\mathrm{LD}} & =\left(\left[s_{\lambda \times A-\theta(X)}, s_{\lambda \times A-\tau(X)}\right],\left(\left(1-\left(1-u_{\mathrm{AMG}}(x)\right)^{\lambda},\left(v_{\mathrm{ANG}}(x)\right)^{\lambda}\right),\left(1-\left(1-\alpha_{\mathrm{AMG}}\right)^{\lambda}, \beta_{\mathrm{ANG}}\right)^{\lambda}\right)\right), \\
A_{\mathrm{LD}}^{\lambda} & =\left(\left[s_{(A-\theta(X))^{\lambda}}, s_{(A-\tau(X))^{\lambda}}\right],\left(\left(u_{\mathrm{AMG}}(x)\right)^{\lambda}, 1-\left(1-\left(v_{\mathrm{ANG}}(x)\right)^{\lambda}\right)\right),\left(\left(\alpha_{\mathrm{AMG}}\right)^{\lambda}, 1-\left(1-\beta_{\mathrm{ANG}}\right)^{\lambda}\right)\right) .
\end{aligned}
$$

Example 1. For any two LDULNs $A_{\mathrm{LD}}=\left(\left[s_{1}, s_{2}\right]\right.$, $(0.5,0.3),(0.5,0.4))$ and $B_{\mathrm{LD}}=\left(\left[s_{2}, s_{3}\right],(0.7,0.5),(0.1\right.$, $0.3)$, with $\lambda=2$, then by using Definition 10 , we get 


$$
\begin{aligned}
A_{\mathrm{LD}} \oplus B_{\mathrm{LD}} & =\left(\left[s_{3}, s_{5}\right],(0.42,0.15),(0.55,0.12)\right), \\
A_{\mathrm{LD}} \otimes B_{\mathrm{LD}} & =\left(\left[s_{2}, s_{6}\right],(0.15,0.42),(0.12,0.55)\right), \\
2 A_{\mathrm{LD}} & =\left(\left[s_{2}, s_{4}\right],(0.75,0.09),(0.75,0.16)\right), \\
A_{\mathrm{LD}}^{2} & =\left(\left[s_{1}, s_{4}\right],(0.09,0.75),(0.16,0.75)\right) .
\end{aligned}
$$

Theorem 1. For any two LDULNs $A_{L D}=\left(\left[s_{A-\theta(X)}\right.\right.$, $\left.\left.s_{A-\tau(X)}\right],\left(u_{A M G}(x), v_{A N G}(x)\right),\left(\alpha_{A M G}, \beta_{A N G}\right)\right)$ and $B_{L D}=$ $\left.\left(\left[s_{B-\theta}(X), s_{B-\tau(X)}\right],\left(u_{B M G}(x)\right), v_{B N G}(x)\right),\left(\alpha_{B M G}, \beta_{B N G}\right)\right)$,

(1) $A_{L D} \oplus B_{L D}=B_{L D} \oplus A_{L D}$

(2) $A_{L D} \otimes B_{L D}=B_{L D} \otimes A_{L D}$

(3) $\lambda\left(A_{L D} \oplus B_{L D}\right)=\lambda A_{L D} \oplus \lambda B_{L D}, \lambda>0$

(4) $\lambda_{1} A_{L D} \oplus \lambda_{2} A_{L D}=\left(\lambda_{1}+\lambda_{2}\right) A_{L D}, \lambda_{1}, \lambda_{2}>0$

(5) $A_{L D}^{\lambda_{1}} \otimes A_{L D}^{\lambda_{2}}=A_{L D}^{\lambda_{1}+\lambda_{2}}, \lambda_{1}, \lambda_{2}>0$

(6) $A_{L D}^{\lambda_{1}} \otimes A_{L D}^{\lambda_{1}}=\left(A_{L D} \otimes B_{L D}\right)^{\lambda_{1}}, \lambda_{1}>0$

Definition 11. For any LDULN $A_{\mathrm{LD}}=\left(\left[s_{A-\theta}\right.\right.$ $\left.\left.(X), s_{A-\tau(X)}\right],\left(u_{\mathrm{AMG}}(x), v_{\mathrm{ANG}}(x)\right),\left(\alpha_{\mathrm{AMG}}, \beta_{\mathrm{ANG}}\right)\right)$, the expected value $(\mathrm{EV})$ is elaborated by

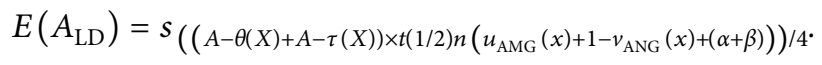

Example 2. For any LDULN $A_{\mathrm{LD}}=\left(\left[s_{1}, s_{2}\right],(0.5\right.$, $0.3),(0.5,0.4))$, the expected value (EV) is elaborated as

$$
E\left(A_{\mathrm{LD}}\right)=s_{((1+2) \times(1 / 2)(0.5+1-0.3+0.5+0.4)) / 4}=s_{((3) \times 1.05) / 4}=s_{0.7875} .
$$

Definition 12. For any LDULN $A_{\mathrm{LD}}=\left(\left[s_{A-\theta(X)}\right.\right.$, $\left.\left.s_{A-\tau(X)}\right],\left(u_{\mathrm{AMG}}(x), v_{\mathrm{ANG}}(x)\right),\left(\alpha_{\mathrm{AMG}}, \beta_{\mathrm{ANG}}\right)\right)$, the accuracy value $(\mathrm{AV})$ is elaborated by

$$
\varsigma_{A_{\mathrm{LD}}}=\varsigma\left(A_{\mathrm{LD}}\right)=s_{\left(\left(u\left(a_{1}\right)+v\left(a_{1}\right)\right) \times(1 / 2)\left(u_{\mathrm{AMG}}(x)+t v_{\mathrm{ANG}} n(x) q+h(\alpha+\beta)\right)\right) / 4} .
$$

Definition 13. For any two LDULNs $A_{\mathrm{LD}}=\left(\left[s_{A-\theta(X)}\right.\right.$, $\left.\left.s_{A-\tau(X)}\right],\left(u_{\mathrm{AMG}}(x), v_{\mathrm{ANG}}(x)\right),\left(\alpha_{\mathrm{AMG}}, \quad \beta_{\mathrm{ANG}}\right)\right)$ and $B_{\mathrm{LD}}=$ $\left(\left[s_{B-\theta}(X), s_{B-\tau(X)}\right],\left(u_{\mathrm{BMG}}(x), v_{\mathrm{BNG}}(x)\right),\left(\alpha_{\mathrm{BMG}}, \beta_{\mathrm{BNG}}\right)\right)$, then

(1) If $\varsigma_{A_{\mathrm{LD} 1}}<\varsigma_{A_{\mathrm{LD} 2}}$, then $A_{\mathrm{LD} 1}<A_{\mathrm{LD} 2}$

(2) If $\varsigma_{A_{\mathrm{LD} 1}}>\varsigma_{A_{\mathrm{LD} 2}}$, then $A_{\mathrm{LD} 1}>A_{\mathrm{LD} 2}$

(3) If $\varsigma_{A_{\mathrm{LD} 1}}=\varsigma_{A_{\mathrm{LD} 2}}$, then
(1) If $\varrho_{A_{\mathrm{LD} 1}}<\varrho_{A_{\mathrm{LD}}}$, then $A_{\mathrm{LD} 1}<A_{\mathrm{LD} 2}$
(2) If $\varrho_{A_{\mathrm{LD} 1}}>\varrho_{A_{\mathrm{LD} 2}}$, then $A_{\mathrm{LD} 1}>A_{\mathrm{LD} 2}$
(3) If $\varrho_{A_{\mathrm{LD} 1}}=\varrho_{A_{\mathrm{LD} 2}}$, then $A_{\mathrm{LD} 1} \approx A_{\mathrm{LD} 2}$

\section{The Power Aggregation (PA) Operator}

Here, we review the basic Einstein t-norm and t-conorm, which are useful for the elaborated approaches.

(1) $a \otimes_{\varepsilon} b=(a+b) /(1+a \cdot b), a, b \in[0,1]$

(2) $a \oplus_{\varepsilon} b=(a \cdot b) /(1+(1-a) \cdot(1-b)), a, b \in[0,1]$

By using any two LDULNs $A_{\mathrm{LD}}=\left(\left[s_{A-\theta(X)}\right.\right.$, $\left.\left.s_{A-\tau(X)}\right],\left(u_{\mathrm{AMG}}(x), v_{\mathrm{ANG}}(x)\right),\left(\alpha_{\mathrm{AMG}}, \beta_{\mathrm{ANG}}\right)\right)$ and $B_{\mathrm{LD}}=$ $\left(\left[s_{B-\theta(X)}, s_{B-\tau(X)}\right],\left(u_{\mathrm{BMG}}(x), v_{\mathrm{BNG}}(x)\right),\left(\alpha_{\mathrm{BMG}}, \beta_{\mathrm{BNG}}\right)\right)$,

$$
\begin{aligned}
& A_{\mathrm{LD}} \otimes_{\varepsilon} B_{\mathrm{LD}}=\left(\left[s_{A-\theta(X) \cdot B-\theta(X)}, s_{A-\tau(X) \cdot B-\tau(X)}\right],\left(\frac{u_{\mathrm{AMG}}(x) \cdot u_{\mathrm{BMG}}(x)}{1+\left(1-u_{\mathrm{AMG}}(x)\right) \cdot\left(1-u_{\mathrm{BNG}}(x)\right)}, \frac{v_{\mathrm{ANG}}(x)+v_{\mathrm{BNG}}(x)}{1+v_{\mathrm{ANG}}(x) \cdot v_{\mathrm{BNG}}(x)}\right),\right. \\
& \left.\cdot\left(\frac{\alpha_{\mathrm{AMG}} \cdot \alpha_{\mathrm{BMG}}}{1+\left(1-\alpha_{\mathrm{AMG}}\right) \cdot\left(1-\alpha_{\mathrm{BMG}}\right)}, \frac{\beta_{\mathrm{ANG}}+\beta_{\mathrm{BNG}}}{1+\beta_{\mathrm{ANG}} \cdot \beta_{\mathrm{BNG}}}\right)\right) \\
& A_{\mathrm{LD}} \otimes_{\varepsilon} B_{\mathrm{LD}}=\left(\left[s_{A-\theta(X)+B-\theta(X)}, s_{A-\tau(X)+B-\tau(X)}\right],\left(\frac{u_{\mathrm{AMG}}(x)+u_{\mathrm{BMG}}(x)}{1+u_{\mathrm{AMG}}(x) \cdot u_{\mathrm{BMG}}(x)}, \frac{v_{\mathrm{ANG}}(x) \cdot v_{\mathrm{BNG}}(x)}{1+\left(1-v_{\mathrm{ANG}}(x)\right) \cdot\left(1-v_{\mathrm{BNG}}(x)\right)}\right),\right. \\
& \left.\cdot\left(\frac{\alpha_{\mathrm{AMG}}+\alpha_{\mathrm{BMG}}}{1+\alpha_{\mathrm{AMG}} \cdot \alpha_{\mathrm{BMG}}}, \frac{\beta_{\mathrm{ANG}} \cdot \beta_{\mathrm{BNG}}}{1+\left(1-\beta_{\mathrm{ANG}}\right) \cdot\left(1-\beta_{\mathrm{BNG}}\right)}\right)\right) \\
& \lambda A_{\mathrm{LD}}=\left(\left[s_{\lambda \cdot A-\theta(X)}, s_{\lambda \cdot A-\tau(X)}\right],\left(\frac{\left(1+u_{\mathrm{AMG}}(x)\right)^{\lambda}-\left(1-u_{\mathrm{AMG}}(x)\right)^{\lambda}}{\left(1+u_{\mathrm{AMG}}(x)\right)^{\lambda}+\left(1-u_{\mathrm{AMG}}(x)\right)^{\lambda}}, \frac{2\left(v_{\mathrm{ANG}}(x)\right)^{\lambda}}{\left(2-v_{\mathrm{ANG}}(x)\right)^{\lambda}+\left(v_{\mathrm{ANG}}(x)\right)^{\lambda}}\right)\right), \\
& \left.\left.\cdot\left(\frac{\left(1+\alpha_{\mathrm{AMG}}\right)^{\lambda}-\left(1-\alpha_{\mathrm{AMG}}\right)^{\lambda}}{\left(1+\alpha_{\mathrm{AMG}}\right)^{\lambda}+\left(1-\alpha_{\mathrm{AMG}}\right)^{\lambda}}, \frac{2\left(\beta_{\mathrm{ANG}}\right)^{\lambda}}{\left(2-\beta_{\mathrm{ANG}}\right)^{\lambda}+\left(\beta_{\mathrm{ANG}}\right)^{\lambda}}\right)\right)\right)
\end{aligned}
$$




$$
\begin{aligned}
& A_{\mathrm{LD}}^{\lambda}=\left(\left[s_{A-\theta(X)^{\lambda}}, s_{A-\tau(X)^{\lambda}}\right],\left(\frac{2\left(u_{\mathrm{AMG}}(x)\right)^{\lambda}}{\left(2-u_{\mathrm{AMG}}(x)\right)^{\lambda}+\left(u_{\mathrm{AMG}}(x)\right)^{\lambda}}\right), \frac{\left(1+v_{\mathrm{ANG}}(x)\right)^{\lambda}-\left(1-v_{\mathrm{ANG}}(x)\right)^{\lambda}}{\left(1+v_{\mathrm{ANG}}(x)\right)^{\lambda}+\left(1-v_{\mathrm{ANG}}(x)\right)^{\lambda}}\right), \\
& \left.\left.\cdot\left(\frac{2\left(\alpha_{\mathrm{AMG}}\right)^{\lambda}}{\left(2-\alpha_{\mathrm{AMG}}\right)^{\lambda}+\left(\alpha_{\mathrm{AMG}}\right)^{\lambda}}\right), \frac{\left(1+\beta_{\mathrm{ANG}}\right)^{\lambda}-\left(1-\beta_{\mathrm{ANG}}\right)^{\lambda}}{\left(1+\beta_{\mathrm{ANG}}\right)^{\lambda}+\left(1-\beta_{\mathrm{ANG}}\right)^{\lambda}}\right)\right) \text {. }
\end{aligned}
$$

Theorem 2. For any two LDULNs $A_{L D}=\left(\left[s_{A-\theta(X)}\right.\right.$, $\left.\left.s_{A-\tau(X)}\right],\left(u_{A M G}(x), v_{A N G}(x)\right),\left(\alpha_{A M G}, \beta_{A N G}\right)\right)$ and $B_{L D}=([s$ $\left.\left.{ }_{B-\theta(X)}, s_{B-\tau(X)}\right],\left(u_{B M G}(x), v_{B N G}(x)\right),\left(\alpha_{B M G}, \beta_{B N G}\right)\right)$, then

(1) $A_{L D} \otimes_{\varepsilon} B_{L D}=B_{L D} \otimes_{\varepsilon} A_{L D}$

(2) $A_{L D} \oplus_{\varepsilon} B_{L D}=B_{L D} \oplus_{\varepsilon} A_{L D}$

(3) $\lambda\left(A_{L D} \otimes_{\varepsilon} B_{L D}\right)=\lambda A_{L D} \otimes_{\varepsilon} \lambda B_{L D}, \lambda>0$
(4) $\lambda_{1} A_{L D} \otimes_{\varepsilon} \lambda_{2} A_{L D}=\left(\lambda_{1}+\lambda_{2}\right) \otimes_{\varepsilon} A_{L D}, \lambda_{1}, \lambda_{2}>0$

(5) $A_{L D}^{\lambda_{1}} \otimes_{\varepsilon} A_{L D}^{\lambda_{2}}=A_{L D}^{\lambda_{1}+\lambda_{2}}, \lambda_{1}, \lambda_{2}>0$

(6) $A_{L D}^{\lambda_{1}} \otimes_{\varepsilon} A_{L D}^{\lambda_{1}}=\left(A_{L D} \otimes_{\varepsilon} B_{L D}\right)^{\lambda_{1}}, \lambda_{1}>0$

Proof. The proof of the first two parts is trivial. Additionally, we prove that part 3 is

$$
\begin{aligned}
A_{\mathrm{LD}} \otimes_{\varepsilon} B_{\mathrm{LD}}= & \left(\left[s_{A-\theta(X)+B-\theta(X)}, s_{A-\tau(X)+B-\tau(X)}\right],\left(\frac{u_{\mathrm{AMG}}(x)+u_{\mathrm{BMG}}(x)}{1+u_{\mathrm{AMG}}(x) \cdot u_{\mathrm{BMG}}(x)}, \frac{v_{\mathrm{ANG}}(x) \cdot v_{\mathrm{BNG}}(x)}{1+\left(1-v_{\mathrm{ANG}}(x)\right) \cdot\left(1-v_{\mathrm{BNG}}(x)\right)}\right)\right. \\
& \left.\cdot\left(\frac{\alpha_{\mathrm{AMG}}+\alpha_{\mathrm{BMG}}}{1+\alpha_{\mathrm{AMG}} \cdot \alpha_{\mathrm{BMG}}}, \frac{\beta_{\mathrm{ANG}} \cdot \beta_{\mathrm{BNG}}}{1+\left(1-\beta_{\mathrm{ANG}}\right) \cdot\left(1-\beta_{\mathrm{BNG}}\right)}\right)\right) ;
\end{aligned}
$$

then,

$$
\begin{aligned}
\lambda\left(A_{\mathrm{LD}} \otimes_{\varepsilon} B_{\mathrm{LD}}\right)= & \left(\left[s_{\lambda(A-\theta(X)+B-\theta(X))}, s_{\lambda(A-\tau(X)+B-\tau(X))}\right],\right. \\
& \left(\frac{\left(\left(1+u_{\mathrm{AMG}}(x)\right)\left(1+u_{\mathrm{BMG}}(x)\right)\right)^{\lambda}-\left(\left(1+u_{\mathrm{AMG}}(x)\right)\left(1+u_{\mathrm{BMG}}(x)\right)\right)^{\lambda}}{\left(\left(1+u_{\mathrm{AMG}}(x)\right)\left(1+u_{\mathrm{BMG}}(x)\right)\right)^{\lambda}+\left(\left(1+u_{\mathrm{AMG}}(x)\right)\left(1+u_{\mathrm{BMG}}(x)\right)\right)^{\lambda}}\right. \\
& \left.\cdot \frac{2\left(v_{\mathrm{ANG}}(x) \cdot v_{\mathrm{BNG}}(x)\right)^{\lambda}}{\left(4-2 v_{\mathrm{ANG}}(x)-2 v_{\mathrm{BNG}}(x)+v_{\mathrm{ANG}}(x) \cdot v_{\mathrm{BNG}}(x)\right)^{\lambda}+\left(v_{\mathrm{ANG}}(x) \cdot v_{\mathrm{BNG}}(x)\right)^{\lambda}}\right), \\
& \left(\frac{\left(\left(1+\alpha_{\mathrm{AMG}}\right)\left(1+\alpha_{\mathrm{BMG}}\right)\right)^{\lambda}-\left(\left(1+\alpha_{\mathrm{AMG}}\right)\left(1+\alpha_{\mathrm{BMG}}\right)\right)^{\lambda}}{\left(\left(1+\alpha_{\mathrm{AMG}}\right)\left(1+\alpha_{\mathrm{BMG}}\right)\right)^{\lambda}+\left(\left(1+\alpha_{\mathrm{AMG}}\right)\left(1+\alpha_{\mathrm{BMG}}\right)\right)^{\lambda}} \frac{2\left(\beta_{\mathrm{ANG}} \cdot \beta_{\mathrm{BNG}}\right)^{\lambda}}{\left.\left.\left(4-2 \beta_{\mathrm{ANG}}-2 \beta_{\mathrm{BNG}}+\beta_{\mathrm{ANG}} \cdot \beta_{\mathrm{BNG}}\right)^{\lambda}+\left(\beta_{\mathrm{ANG}} \cdot \beta_{\mathrm{BNG}}\right)^{\lambda}\right)\right) .}\right.
\end{aligned}
$$

For the right hand of the part (3),

$$
\begin{aligned}
\lambda A_{\mathrm{LD}}= & \left(\left[s_{\lambda \cdot A-\theta(X)}, s_{\lambda \cdot A-\tau(X)}\right],\left(\frac{\left(1+u_{\mathrm{AMG}}(x)\right)^{\lambda}-\left(1-u_{\mathrm{AMG}}(x)\right)^{\lambda}}{\left(1+u_{\mathrm{AMG}}(x)\right)^{\lambda}+\left(1-u_{\mathrm{AMG}}(x)\right)^{\lambda}}, \frac{2\left(v_{\mathrm{ANG}}(x)\right)^{\lambda}}{\left(2-v_{\mathrm{ANG}}(x)\right)^{\lambda}+\left(v_{\mathrm{ANG}}(x)\right)^{\lambda}}\right)\right. \\
& \left.\cdot\left(\frac{\left(1+\alpha_{\mathrm{AMG}}\right)^{\lambda}-\left(1-\alpha_{\mathrm{AMG}}\right)^{\lambda}}{\left(1+\alpha_{\mathrm{AMG}}\right)^{\lambda}+\left(1-\alpha_{\mathrm{AMG}}\right)^{\lambda}} \frac{2\left(\beta_{\mathrm{ANG}}\right)^{\lambda}}{\left(2-\beta_{\mathrm{ANG}}\right)^{\lambda}+\left(\beta_{\mathrm{ANG}}\right)^{\lambda}}\right)\right),
\end{aligned}
$$




$$
\begin{aligned}
\lambda B_{\mathrm{LD}}= & \left(\left[s_{\lambda \cdot B-\theta(X)}, s_{\lambda \cdot B-\tau(X)}\right],\left(\frac{\left(1+u_{\mathrm{BMG}}(x)\right)^{\lambda}-\left(1-u_{\mathrm{BMG}}(x)\right)^{\lambda}}{\left(1+u_{\mathrm{BMG}}(x)\right)^{\lambda}+\left(1-u_{\mathrm{BMG}}(x)\right)^{\lambda}}, \frac{2\left(v_{\mathrm{BNG}}(x)\right)^{\lambda}}{\left(2-v_{\mathrm{BNG}}(x)\right)^{\lambda}+\left(v_{\mathrm{BNG}}(x)\right)^{\lambda}}\right)\right) \\
& \left.\left.\cdot\left(\frac{\left(1+\alpha_{\mathrm{BMG}}\right)^{\lambda}-\left(1-\alpha_{\mathrm{BMG}}\right)^{\lambda}}{\left(1+\alpha_{\mathrm{BMG}}\right)^{\lambda}+\left(1-\alpha_{\mathrm{BMG}}\right)^{\lambda}} \frac{2\left(\beta_{\mathrm{BNG}}\right)^{\lambda}}{\left(2-\beta_{\mathrm{BNG}}\right)^{\lambda}+\left(\beta_{\mathrm{BNG}}\right)^{\lambda}}\right)\right)\right) .
\end{aligned}
$$

Then,

$$
\begin{aligned}
\lambda\left(A_{\mathrm{LD}} \otimes_{\varepsilon} B_{\mathrm{LD}}\right)= & \left(\left[s_{\left.\lambda(A-\theta(X)+B-\theta(X)), s_{\lambda(A-\tau(X)+B-\tau(X))}\right]}\right.\right. \\
& \left(\frac{\left(\left(1+u_{\mathrm{AMG}}(x)\right)\left(1+u_{\mathrm{BMG}}(x)\right)\right)^{\lambda}-\left(\left(1+u_{\mathrm{AMG}}(x)\right)\left(1+u_{\mathrm{BMG}}(x)\right)\right)^{\lambda}}{\left(\left(1+u_{\mathrm{AMG}}(x)\right)\left(1+u_{\mathrm{BMG}}(x)\right)\right)^{\lambda}+\left(\left(1+u_{\mathrm{AMG}}(x)\right)\left(1+u_{\mathrm{BMG}}(x)\right)\right)^{\lambda}}\right. \\
& \left.\cdot \frac{2\left(v_{\mathrm{ANG}}(x) \cdot v_{\mathrm{BNG}}(x)\right)^{\lambda}}{\left(4-2 v_{\mathrm{ANG}}(x)-2 v_{\mathrm{BNG}}(x)+v_{\mathrm{ANG}}(x) \cdot v_{\mathrm{BNG}}(x)\right)^{\lambda}+\left(v_{\mathrm{ANG}}(x) \cdot v_{\mathrm{BNG}}(x)\right)^{\lambda}}\right), \\
( & \left(\left(1+\alpha_{\mathrm{AMG}}\right)\left(1+\alpha_{\mathrm{BMG}}\right)\right)^{\lambda}-\left(\left(1+\alpha_{\mathrm{AMG}}\right)\left(1+\alpha_{\mathrm{BMG}}\right)\right)^{\lambda} \\
\left(\left(1+\alpha_{\mathrm{AMG}}\right)\left(1+\alpha_{\mathrm{BMG}}\right)\right)^{\lambda}+\left(\left(1+\alpha_{\mathrm{AMG}}\right)\left(1+\alpha_{\mathrm{BMG}}\right)\right)^{\lambda} & \left.\left.\frac{2\left(\beta_{\mathrm{ANG}} \cdot \beta_{\mathrm{BNG}}\right)^{\lambda}}{\left(4-2 \beta_{\mathrm{ANG}}-2 \beta_{\mathrm{BNG}}+\beta_{\mathrm{ANG}} \cdot \beta_{\mathrm{BNG}}\right)^{\lambda}+\left(\beta_{\mathrm{ANG}} \cdot \beta_{\mathrm{BNG}}\right)^{\lambda}}\right)\right) .
\end{aligned}
$$

Hence, we get $\lambda\left(A_{\mathrm{LD}} \otimes_{\varepsilon} B_{\mathrm{LD}}\right)=\lambda A_{\mathrm{LD}} \otimes_{\varepsilon} \lambda B_{\mathrm{LD}}, \lambda>0$. Similarly, we prove that part 4 . Know, for part (5), we have

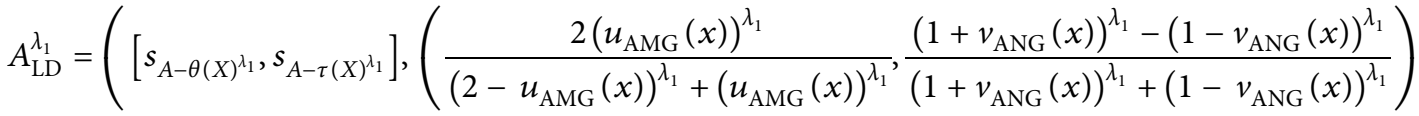

$$
\begin{aligned}
& \left.\left.\cdot\left(\frac{2\left(\alpha_{\mathrm{AMG}}\right)^{\lambda_{1}}}{\left(2-\alpha_{\mathrm{AMG}}\right)^{\lambda_{1}}+\left(\alpha_{\mathrm{AMG}}\right)^{\lambda_{1}}}\right), \frac{\left(1+\beta_{\mathrm{ANG}}\right)^{\lambda_{1}}-\left(1-\beta_{\mathrm{ANG}}\right)^{\lambda_{1}}}{\left(1+\beta_{\mathrm{ANG}}\right)^{\lambda_{1}}+\left(1-\beta_{\mathrm{ANG}}\right)^{\lambda_{1}}}\right)\right)
\end{aligned}
$$

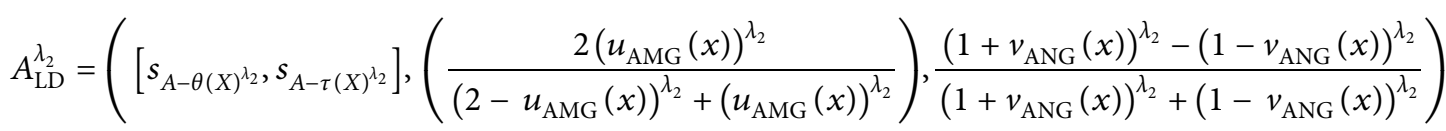

$$
\begin{aligned}
& \left.\left.\cdot\left(\frac{2\left(\alpha_{\mathrm{AMG}}\right)^{\lambda_{2}}}{\left(2-\alpha_{\mathrm{AMG}}\right)^{\lambda_{2}}+\left(\alpha_{\mathrm{AMG}}\right)^{\lambda_{2}}}\right), \frac{\left(1+\beta_{\mathrm{ANG}}\right)^{\lambda_{2}}-\left(1-\beta_{\mathrm{ANG}}\right)^{\lambda_{2}}}{\left(1+\beta_{\mathrm{ANG}}\right)^{\lambda_{2}}+\left(1-\beta_{\mathrm{ANG}}\right)^{\lambda_{2}}}\right)\right) .
\end{aligned}
$$

Then, 


$$
\begin{aligned}
A_{\mathrm{LD}}^{\lambda_{1}} \otimes_{E} A_{\mathrm{LD}}^{\lambda_{2}}= & \left(\left[S_{A-\theta(X)} \lambda_{1}+\lambda_{2}, S_{A-\tau(X)} \lambda_{1}+\lambda_{2}\right]\right. \\
& \left(\frac{\left(u_{\mathrm{AMG}}(x)\right)^{\lambda_{1}+\lambda_{2}}}{\left(2-u_{\mathrm{AMG}}(x)\right)^{\lambda_{2}}\left(u_{\mathrm{AMG}}(x)\right)^{\lambda_{1}}+\left(2-u_{\mathrm{AMG}}(x)\right)^{\lambda_{1}}\left(u_{\mathrm{AMG}}(x)\right)^{\lambda_{2}}},\right. \\
& \left.\cdot \frac{2\left(1+v_{\mathrm{ANG}}(x)^{\lambda_{1}+\lambda_{2}}\right)}{\left(\left(1+v_{\mathrm{ANG}}(x)\right)^{\lambda_{1}}+\left(1-v_{\mathrm{ANG}}(x)\right)^{\lambda_{1}}\right)\left(\left(1+v_{\mathrm{ANG}}(x)\right)^{\lambda_{2}}+\left(1-v_{\mathrm{ANG}}(x)\right)^{\lambda_{2}}\right)}\right), \\
& \left.\left.\left(\frac{\left(\alpha_{\mathrm{ANG}}\right)^{\lambda_{1}+\lambda_{2}}}{\left(2-\alpha_{\mathrm{AMG}}\right)^{\lambda_{2}}\left(\alpha_{\mathrm{AMG}}\right)^{\lambda_{1}}+\left(2-\alpha_{\mathrm{AMG}}\right)^{\lambda_{1}}\left(\alpha_{\mathrm{AMG}}\right)^{\lambda_{2}}}\right), \frac{2\left(1+\beta_{\mathrm{ANG}}\right)^{\lambda_{1}+\lambda_{2}}}{\left(\left(1+\beta_{\mathrm{ANG}}\right)^{\lambda_{1}}+\left(1+\beta_{\mathrm{ANG}}\right)^{\lambda_{1}}\right)\left(\left(1+\beta_{\mathrm{ANG}}\right)^{\lambda_{2}}+\left(1+\beta_{\mathrm{ANG}}\right)^{\lambda_{2}}\right)^{\prime}}\right)\right) .
\end{aligned}
$$

For the right hand of the part (5), we get

$$
\begin{aligned}
& A_{\mathrm{LD}}^{\lambda_{1}+\lambda_{2}}=\left(\left[S_{A-\theta(X)} \lambda_{1}+\lambda_{2}, S_{A-\tau(X)} \lambda_{1}+\lambda_{2}\right]\right. \\
& \left(\frac{\left(u_{\mathrm{AMG}}(x)\right)^{\lambda_{1}+\lambda_{2}}}{\left(2-u_{\mathrm{AMG}}(x)\right)^{\lambda_{2}}\left(u_{\mathrm{AMG}}(x)\right)^{\lambda_{1}}+\left(2-u_{\mathrm{AMG}}(x)\right)^{\lambda_{1}}\left(u_{\mathrm{AMG}}(x)\right)^{\lambda_{2}}},\right. \\
& \left.\cdot \frac{2\left(1+v_{\mathrm{ANG}}(x)^{\lambda_{1}+\lambda_{2}}\right)}{\left(\left(1+v_{\mathrm{ANG}}(x)\right)^{\lambda_{1}}+\left(1-v_{\mathrm{ANG}}(x)\right)^{\lambda_{1}}\right)\left(\left(1+v_{\mathrm{ANG}}(x)\right)^{\lambda_{2}}+\left(1-v_{\mathrm{ANG}}(x)\right)^{\lambda_{2}}\right)}\right), \\
& \left(\frac{\left(\alpha_{\mathrm{ANG}}\right)^{\lambda_{1}+\lambda_{2}}}{\left(2-\alpha_{\mathrm{AMG}}\right)^{\lambda_{2}}\left(\alpha_{\mathrm{AMG}}\right)^{\lambda_{1}}+\left(2-\alpha_{\mathrm{AMG}}\right)^{\lambda_{1}}\left(\alpha_{\mathrm{AMG}}\right)^{\lambda_{2}}}, \frac{2\left(1+\beta_{\mathrm{ANG}}\right)^{\lambda_{1}+\lambda_{2}}}{\left(\left(1+\beta_{\mathrm{ANG}}\right)^{\lambda_{1}}+\left(1+\beta_{\mathrm{ANG}}\right)^{\lambda_{1}}\right)\left(\left(1+\beta_{\mathrm{ANG}}\right)^{\lambda_{2}}+\left(1+\beta_{\mathrm{ANG}}\right)^{\lambda_{2}}\right)^{\prime}},\right) .
\end{aligned}
$$

Hence, we get $A_{\mathrm{LD}}^{\lambda_{1}} \otimes_{\varepsilon} A_{\mathrm{LD}}^{\lambda_{2}}=A_{\mathrm{LD}}^{\lambda_{1}+\lambda_{2}}, \lambda_{1}, \lambda_{2}>0$. Similarly, we prove that part (6).

\section{Some LDULFPE Operators}

In this section, we combine the PA operator and Einstein operations to the LDUL environment, propose the LDULFPEA operator, LDLFPEWA operator, LDULFPEG operator, and LDULFPEWG operator, and discuss the properties of them.

Definition 14. For any LDULNs $A_{\mathrm{LD}-i}=\left(\left[s_{A-\theta_{i}}, s_{A-\tau_{i}}\right]\right.$, $\left.\left(u_{\mathrm{AMG}-i}, v_{\mathrm{ANG}-i}\right),\left(\alpha_{\mathrm{AMG}-i}, \beta_{\mathrm{ANG}-i}\right)\right), i=1,2, \ldots, n$, the LDULFPEA operator is elaborated by

$$
\begin{gathered}
\operatorname{LDULFPEA}\left(A_{\mathrm{LD}-1}, A_{\mathrm{LD}-2}, \ldots, A_{\mathrm{LD}-n}\right) \\
=\frac{\otimes_{\varepsilon i=1}^{n}\left(1+T\left(A_{\mathrm{LD}-i}\right)\right) * A_{\mathrm{LD}-i}}{\sum_{i=1}^{n}\left(1+T\left(A_{\mathrm{LD}-i}\right)\right)} \\
=\otimes_{\varepsilon i=1}^{n}\left(\frac{\left(1+T\left(A_{\mathrm{LD}-i}\right)\right) * A_{\mathrm{LD}-i}}{\sum_{i=1}^{n}\left(1+T\left(A_{\mathrm{LD}-i}\right)\right)}\right)
\end{gathered}
$$

where $T\left(A_{\mathrm{LD}-i}\right)=\sum_{j=1}^{n} \sup \left(A_{\mathrm{LD}-i}, A_{\mathrm{LD}-j}\right)$, and $\sup \left(A_{\mathrm{LD}-i}\right.$, $\left.A_{\mathrm{LD}-j}\right)$ is the support for $A_{\mathrm{LD}-i}$ from $A_{\mathrm{LD}-j}$.

Theorem 3. For any LDULFNs $A_{L D-i}=\left(\left[s_{A-} \theta_{i}, s_{A-\tau_{i}}\right]\right.$, $\left.\left(u_{A M G-i}, v_{A N G-i}\right),\left(\alpha_{A M G-i}, \beta_{A N G-i}\right)\right), i=1,2, \ldots, n$, then the result aggregated from Definition 14 is still an LDULFN: 
$\operatorname{LDULFPEA}\left(A_{\mathrm{LD}-1}, A_{\mathrm{LD}-2}, \ldots, A_{\mathrm{LD}-D}\right)=\left(\left[S_{\sum_{i=1}^{n}\left(\left(A \mid \theta_{i}\left(1+T\left(A_{\mathrm{LD}-i}\right)\right)\right) / \sum_{i=1}^{n}\left(1+T\left(A_{\mathrm{LD}-i}\right)\right)\right)}, S_{\left.\sum_{i=1}^{n}\left(A-\tau_{i}\left(\theta_{i}\left(1+T\left(A_{\mathrm{LD}-i}\right)\right)\right) / \sum_{i=1}^{n}\left(1+T\left(A_{\mathrm{LD}-i}\right)\right)\right)\right]}\right.\right.$, $\left(\frac{\prod_{i=1}^{n}\left(1+u_{\mathrm{AMG}-i}\right)\left(\left(1+T\left(A_{\mathrm{LD}-i}\right)\right) / \sum_{i=1}^{n}\left(1+T\left(A_{\mathrm{LD}-i}\right)\right)\right)-\prod_{i=1}^{n}\left(1-u_{\mathrm{AMG}-i}\right)\left(\left(1+T\left(A_{\mathrm{LD}-i}\right)\right) / \sum_{i=1}^{n}\left(1+T\left(A_{\mathrm{LD}-i}\right)\right)\right)}{\prod_{i=1}^{n}\left(1+u_{\mathrm{AMG}-i}\right)\left(\left(1+T\left(A_{\mathrm{LD}-i}\right)\right) / \sum_{i=1}^{n}\left(1+T\left(A_{\mathrm{LD}-i}\right)\right)\right)+\prod_{i=1}^{n}\left(1-u_{\mathrm{AMG}-i}\right)\left(\left(1+T\left(A_{\mathrm{LD}-i}\right)\right) / \sum_{i=1}^{n}\left(1+T\left(A_{\mathrm{LD}-i}\right)\right)\right)}\right.$, $\left.\frac{2 \prod_{i=1}^{n}\left(v_{\mathrm{ANG}-i}\right)\left(\left(1+T\left(A_{\mathrm{LD}-i}\right)\right) / \sum_{i=1}^{n}\left(1+T\left(A_{\mathrm{LD}-i}\right)\right)\right)}{\prod_{i=1}^{n}\left(2-v_{\mathrm{ANG}-i}\right)\left(\left(1+T\left(A_{\mathrm{LD}-i}\right)\right) / \sum_{i=1}^{n}\left(1+T\left(A_{\mathrm{LD}-i}\right)\right)\right)+\prod_{i=1}^{n}\left(v_{\mathrm{ANG}-i}\right)\left(\left(1+T\left(A_{\mathrm{LD}-i}\right)\right) / \sum_{i=1}^{n}\left(1+T\left(A_{\mathrm{LD}-i}\right)\right)\right)}\right)$,

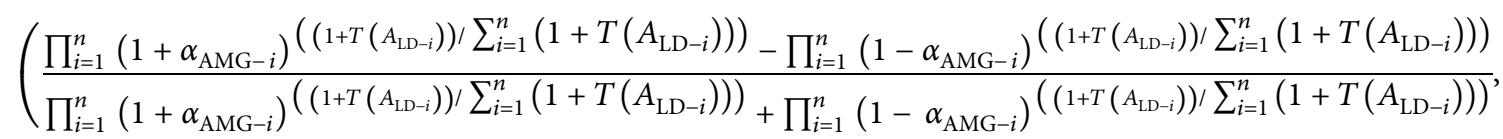

$\left.\left.\frac{2 \prod_{i=1}^{n}\left(\beta_{\mathrm{ANG}-i}\right)^{\left(1+T\left(A_{\mathrm{LD}-i}\right) / \sum_{i=1}^{n}\left(1+T\left(A_{\mathrm{LD}-i}\right)\right)\right)}}{\prod_{i=1}^{n}\left(2-\beta_{\mathrm{ANG}-i}\right)\left(\left(1+T\left(A_{\mathrm{LD}-i}\right)\right) / \sum_{i=1}^{n}\left(1+T\left(A_{\mathrm{LD}-i}\right)\right)\right)+\prod_{i=1}^{n}\left(\beta_{\mathrm{ANG}-i}\right)\left(\left(1+T\left(A_{\mathrm{LD}-i}\right)\right) / \sum_{i=1}^{n}\left(1+T\left(A_{\mathrm{LD}-i}\right)\right)\right)}\right)\right)$,

where $T\left(A_{L D-i}\right)=\sum_{\substack{j=1 \\ j \neq i}}^{n} \sup \left(A_{L D-i}, A_{L D-j}\right)$, and sup Proof. To simplify equation (26), we suppose $\left(A_{L D-i}, A_{L D-j}\right)$ is the support for $A_{L D-i}$ from $A_{L D-j}$.

$$
\begin{aligned}
& \operatorname{LDULFPEA}\left(A_{\mathrm{LD}-1}, A_{\mathrm{LD}-2}, \ldots, A_{\mathrm{LD}-D}\right)=\left(\left[S_{\sum_{i=1}^{n} A=\theta_{i} c_{i}}, S_{\sum_{i=1}^{n} A-\tau_{i} c_{i}}\right]\right. \\
& \left(\frac{\prod_{i=1}^{n}\left(1+u_{\mathrm{AMG}-i}\right)^{c_{i}}-\prod_{i=1}^{n}\left(1-u_{\mathrm{AMG}-i}\right)^{c_{i}}}{\prod_{i=1}^{n}\left(1+u_{\mathrm{AMG}-i}\right)^{c_{i}}+\prod_{i=1}^{n}\left(1-u_{\mathrm{AMG}-i}\right)^{c_{i}}}, \frac{2 \prod_{i=1}^{n}\left(v_{\mathrm{ANG}-i}\right)^{c_{i}}}{\prod_{i=1}^{n}\left(2-v_{\mathrm{ANG}-i}\right)^{c_{i}}+\prod_{i=1}^{n}\left(v_{\mathrm{ANG}-i}\right)^{c_{i}}}\right), \\
& \left.\left(\frac{\prod_{i=1}^{n}\left(1+\alpha_{\mathrm{AMG}-i}\right)^{c_{i}}-\prod_{i=1}^{n}\left(1-\alpha_{\mathrm{AMG}-i}\right)^{c_{i}}}{\prod_{i=1}^{n}\left(1+\alpha_{\mathrm{AMG}-i}\right)^{c_{i}}+\prod_{i=1}^{n}\left(1-\alpha_{\mathrm{AMG}-i}\right)^{c_{i}}}, \frac{2 \prod_{i=1}^{n}\left(\beta_{\mathrm{ANG}-i}\right)^{c_{i}}}{\prod_{i=1}^{n}\left(2-\beta_{\mathrm{ANG}-i}\right)^{c_{i}}+\prod_{i=1}^{n}\left(\beta_{\mathrm{ANG}-i}\right)^{c_{i}}}\right)\right) .
\end{aligned}
$$

The equation (26) can be proved by mathematical in-

(2) Suppose when $n=k$, equation (26) is right, i.e., duction on $n$ as

(1) When $n=1$, equation (26) is right obviously

$$
\begin{aligned}
& \operatorname{LDULFPEA}\left(A_{\mathrm{LD}-1}, A_{\mathrm{LD}-2}, \ldots, A_{\mathrm{LD}-k}\right)=\left(\left[S_{\sum_{i=1}^{k} A=\theta_{i} c_{i}} S_{\sum_{i=1}^{k} A-\tau_{i} c_{i}}\right]\right. \\
& \left(\frac{\prod_{i=1}^{k}\left(1+u_{\mathrm{AMG}-i}\right)^{c_{i}}-\prod_{i=1}^{k}\left(1-u_{\mathrm{AMG}-i}\right)^{c_{i}}}{\prod_{i=1}^{k}\left(1+u_{\mathrm{AMG}-i}\right)^{c_{i}}+\prod_{i=1}^{k}\left(1-u_{\mathrm{AMG}-i}\right)^{c_{i}}}, \frac{2 \prod_{i=1}^{k}\left(v_{\mathrm{ANG}-i}\right)^{c_{i}}}{\prod_{i=1}^{k}\left(2-v_{\mathrm{ANG}-i}\right)^{c_{i}}+\prod_{i=1}^{k}\left(v_{\mathrm{ANG}-i}\right)^{c_{i}}}\right) \\
& \left.\left(\frac{\prod_{i=1}^{k}\left(1+\alpha_{\mathrm{AMG}-i}\right)^{c_{i}}-\prod_{i=1}^{k}\left(1-\alpha_{\mathrm{AMG}-i}\right)^{c_{i}}}{\prod_{i=1}^{k}\left(1+\alpha_{\mathrm{AMG}-i}\right)^{c_{i}}+\prod_{i=1}^{k}\left(1-\alpha_{\mathrm{AMG}-i}\right)^{c_{i}}} \frac{2 \prod_{i=1}^{k}\left(\beta_{\mathrm{ANG}-i}\right)^{c_{i}}}{\prod_{i=1}^{k}\left(2-\beta_{\mathrm{ANG}-i}\right)^{c_{i}}+\prod_{i=1}^{k}\left(\beta_{\mathrm{ANG}-i}\right)^{c_{i}}}\right)\right)
\end{aligned}
$$

Then, when $n=k+1$, we have 


$$
\begin{aligned}
& \operatorname{LDULFPEA}\left(A_{\mathrm{LD}-1}, A_{\mathrm{LD}-2}, \ldots, A_{\mathrm{LD}-k+1}\right)=\operatorname{LDULFPEA}\left(A_{\mathrm{LD}-1}, A_{\mathrm{LD}-2}, \ldots, A_{\mathrm{LD}-k}\right) \otimes_{\varepsilon}\left(c_{k+1} A_{\mathrm{LD}-i}\right) \\
& =\operatorname{LDULFPEA}\left(A_{\mathrm{LD}-1}, A_{\mathrm{LD}-2}, \ldots, A_{\mathrm{LD}-k}\right) \otimes_{\varepsilon}\left(\left[S_{A-\theta_{k+1} c_{k+1}}, S_{A-\tau_{k+1} c_{k+1}}\right]\right. \\
& \left(\frac{\left(1+u_{\mathrm{AMG}-k+1}\right)^{c_{k+1}}-\left(1-u_{\mathrm{AMG}-k+1}\right)^{c_{k+1}}}{\left(1+u_{\mathrm{AMG}-k+1}\right)^{c_{k+1}}+\left(1-u_{\mathrm{AMG}-k+1}\right)^{c_{k+1}}}, \frac{2\left(v_{\mathrm{ANG}-k+1}\right)^{k+1}}{\left(2-v_{\mathrm{ANG}-k+i}\right)^{k+1}+\left(v_{\mathrm{ANG}-k+i}\right)^{k+1}}\right), \\
& \left.\left(\frac{\left(1+\alpha_{\mathrm{AMG}-k+1}\right)^{c_{k+1}}-\left(1-\alpha_{\mathrm{AMG}-k+1}\right)^{c_{k+1}}}{\left(1+\alpha_{\mathrm{AMG}-k+1}\right)^{c_{k+1}}+\left(1-\alpha_{\mathrm{AMG}-k+1}\right)^{c_{k+1}}}, \frac{2\left(\beta_{\mathrm{ANG}-k+1}\right)^{K+1}}{2-\beta_{\mathrm{ANG}-k+1}^{K+1}+\left(\beta_{\mathrm{ANG}-k+1}\right)^{K+1}}\right)\right) \\
& =\operatorname{LDULFPEA}\left(A_{L D-1}, A_{L D-2}, \ldots, A_{L D-k+1}\right)=\left(\left[S_{\sum_{i=1}^{k+1} A=\theta_{i} c_{i}}, S_{\sum_{i=1}^{k+1} A-\tau_{i} c_{i}}\right]\right. \\
& \left(\frac{\prod_{i=1}^{k+1}\left(1+u_{\mathrm{AMG}-i}\right)^{c_{i}}-\prod_{i=1}^{k+1}\left(1-u_{\mathrm{AMG}-i}\right)^{c_{i}}}{\prod_{i=1}^{k+1}\left(1+u_{\mathrm{AMG}-i}\right)^{c_{i}}+\prod_{i=1}^{k+1}\left(1-u_{\mathrm{AMG}-i}\right)^{c_{i}}}, \frac{2 \prod_{i=1}^{k+1}\left(v_{\mathrm{ANG}-i}\right)^{c_{i}}}{\prod_{i=1}^{k+1}\left(2-v_{\mathrm{ANG}-i}\right)^{c_{i}}+\prod_{i=1}^{k+1}\left(v_{\mathrm{ANG}-i}\right)^{c_{i}}}\right), \\
& \left.\left(\frac{\prod_{i=1}^{k+1}\left(1+\alpha_{\mathrm{AMG}-i}\right)^{c_{i}}-\prod_{i=1}^{k+1}\left(1-\alpha_{\mathrm{AMG}-i}\right)^{c_{i}}}{\prod_{i=1}^{k+1}\left(1+\alpha_{\mathrm{AMG}-i}\right)^{c_{i}}+\prod_{i=1}^{k+1}\left(1-\alpha_{\mathrm{AMG}-i}\right)^{c_{i}}}, \frac{2 \prod_{i=1}^{k+1}\left(\beta_{\mathrm{ANG}-i}\right)^{c_{i}}}{\prod_{i=1}^{k+1}\left(2-\beta_{\mathrm{ANG}-i}\right)^{c_{i}}+\prod_{i=1}^{k+1}\left(\beta_{\mathrm{ANG}-i}\right)^{c_{i}}}\right)\right) .
\end{aligned}
$$

So, when $n=k+1$, equation (26) is also right. According

$$
\operatorname{LDULFPEA}\left(A_{\mathrm{LD}-1}, A_{\mathrm{LD}-2}, \ldots, A_{\mathrm{LD}-n}\right)=A \text {. }
$$
to steps (1) and (2), we can get that equation (26) is right for $n$.

Theorem 4. For any LDULNs $A_{L D-j}=A, j=1,2, \ldots, n$ and $A=\left(\left[s_{A-\theta}, s_{A-\tau}\right],\left(u_{A M G}, v_{A N G}\right),\left(\alpha_{A M G}, \beta_{A N G}\right)\right)$,

Proof. Since $A_{\mathrm{LD}-i}=A$, for all $i$, we have

$\operatorname{LDULFPEA}\left(A_{\mathrm{LD}-1}, A_{\mathrm{LD}-2}, \ldots, A_{\mathrm{LD}-n}\right)=\left(\left[S_{\sum_{i=1}^{n} A \mid \theta_{i}(1+T(A)) /} \sum_{i=1}^{n}(1+T(A)), S_{\sum_{i=1}^{n}\left(A-\tau_{i}\left(\theta_{i}(1+T(A))\right)\right) / \sum_{i=1}^{n}(1+T(A))}\right]\right.$,

$$
\begin{aligned}
& \left(\frac{\prod_{i=1}^{n}\left(1+u_{\mathrm{AMG}}\right)^{(1+T(A)) / \sum_{i=1}^{n}(1+T(A))}-\prod_{i=1}^{n}\left(1-u_{\mathrm{AMG}}\right)^{(1+T(A)) / \sum_{i=1}^{n}(1+T(A))}}{\prod_{i=1}^{n}\left(1+u_{\mathrm{AMG}}\right)^{(1+T(A)) / \sum_{i=1}^{n}(1+T(A))}+\prod_{i=1}^{n}\left(1-u_{\mathrm{AMG}}\right)^{(1+T(A)) / \sum_{i=1}^{n}(1+T(A))}},\right. \\
& \left.\cdot \frac{2 \prod_{i=1}^{n}\left(v_{\mathrm{ANG}}\right)^{(1+T(A)) /} \sum_{i=1}^{n}(1+T(A))}{\left.\prod_{i=1}^{n}\left(2-v_{\mathrm{ANG}}\right)^{(1+T(A)) / \sum_{i=1}^{n}(1+T(A))}+\prod_{i=1}^{n}\left(v_{\mathrm{ANG}}\right)^{(1+T(A)) / \sum_{i=1}^{n}(1+T(A))}\right)}\right) \\
& \left(\frac{\prod_{i=1}^{n}\left(1+\alpha_{\mathrm{AMG}}\right)^{(1+T(A)) / \sum_{i=1}^{n}(1+T(A))}-\prod_{i=1}^{n}\left(1-\alpha_{\mathrm{AMG}}\right)^{(1+T(A)) / \sum_{i=1}^{n}(1+T(A))}}{\prod_{i=1}^{n}\left(1+\alpha_{\mathrm{AMG}}\right)^{(1+T(A)) / \sum_{i=1}^{n}(1+T(A))}+\prod_{i=1}^{n}\left(1-\alpha_{\mathrm{AMG}}\right)^{(1+T(A)) / \sum_{i=1}^{n}(1+T(A))}},\right. \\
& \cdot \frac{2 \prod_{i=1}^{n}\left(\beta_{\mathrm{ANG}}\right)^{(1+T(A)) / \sum_{i=1}^{n}(1+T(A))}}{\left.\left.\prod_{i=1}^{n}\left(2-\beta_{\mathrm{ANG}}\right)^{(1+T(A)) /} \sum_{i=1}^{n}(1+T(A))+\prod_{i=1}^{n}\left(\beta_{\mathrm{ANG}}\right)^{(1+T(A)) / \sum_{i=1}^{n}(1+T(A))}\right)\right)}
\end{aligned}
$$




$$
\begin{aligned}
& =\left(\left[S_{(A-\theta)} \sum_{i=1}^{n}(1+T(A)) / \sum_{i=1}^{n}(1+T(A)), S_{(A-\tau)} \sum_{i=1}^{n}(1+T(A)) / \sum_{i=1}^{n}(1+T(A))\right],\right. \\
& \left(\frac{\left(1+u_{\mathrm{AMG}}\right)^{(1+T(A)) / \sum_{i=1}^{n}(1+T(A))}-\left(1-u_{\mathrm{AMG}}\right)^{(1+T(A)) / \sum_{i=1}^{n}(1+T(A))}}{\left(1+u_{\mathrm{AMG}}\right)^{(1+T(A)) / \sum_{i=1}^{n}(1+T(A))}+\left(1-u_{\mathrm{AMG}}\right)^{(1+T(A)) / \sum_{i=1}^{n}(1+T(A))},}\right. \\
& \cdot \frac{2\left(v_{\mathrm{ANG}}\right)^{(1+T(A)) / \sum_{i=1}^{n}(1+T(A))}}{\left.2-\left(v_{\mathrm{ANG}}\right)^{(1+T(A)) / \sum_{i=1}^{n}(1+T(A))}+\left(v_{\mathrm{ANG}}\right)^{(1+T(A)) / \sum_{i=1}^{n}(1+T(A))}\right)},
\end{aligned}
$$

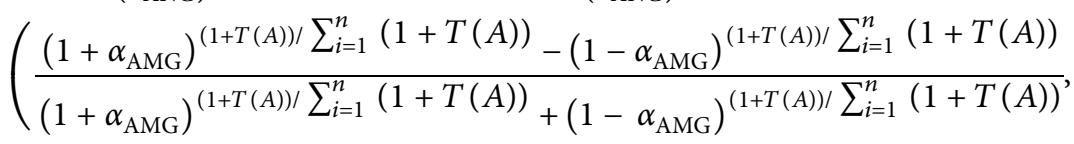

$$
\begin{aligned}
& \left.\cdot \frac{2\left(\beta_{\mathrm{ANG}}\right)^{(1+T(A)) / \sum_{i=1}^{n}(1+T(A))}}{\left(\left(2+\beta_{\mathrm{ANG}}\right)^{(1+T(A)) / \sum_{i=1}^{n}(1+T(A))}\right)+\left(\beta_{\mathrm{ANG}}\right)^{(1+T(A)) / \sum_{i=1}^{n}(1+T(A))}}\right) \\
& =\left(\left[s_{A-\theta}, s_{A-\tau}\right],\left(u_{\mathrm{AMG}}, v_{\mathrm{ANG}}\right),\left(\alpha_{\mathrm{AMG}}, \beta_{\mathrm{ANG}}\right)\right)=A \text {. }
\end{aligned}
$$

Theorem 5. For any LDULNs $A_{L D-i}=\left(\left[s_{A-}\right.\right.$ $\left.\left.\theta_{i}, s_{A-\tau_{i}}\right],\left(u_{A M G-i}, v_{A N G-i}\right),\left(\alpha_{A M G-i}, \beta_{A N G-i}\right)\right), i=1,2, \ldots, n$, if LDULFPEA operator lies between the max and min operators,

$$
\begin{aligned}
& \min \left(A_{\mathrm{LD}-1}, A_{\mathrm{LD}-2}, \ldots, A_{\mathrm{LD}-n}\right)=\min A, \\
& \max \left(A_{\mathrm{LD}-1}, A_{\mathrm{LD}-2}, \ldots, A_{\mathrm{LD}-n}\right)=\max A ;
\end{aligned}
$$

then,

$$
\min A \leq \operatorname{LDULFPEA}\left(A_{\mathrm{LD}-1}, A_{\mathrm{LD}-2}, \ldots, A_{\mathrm{LD}-n}\right) \leq \max A .
$$

Proof. First, let $g\left(u_{\mathrm{AMG}-i}\right)=\left(1-\left(u_{\mathrm{AMG}-i}\right)\right) /\left(1+\left(u_{\mathrm{AMG}-i}\right)\right)$, $\left(u_{\mathrm{AMG}-i}\right) \in[0,1]$, we can get $g^{\prime}\left(u_{\mathrm{AMG}-i}\right)=-2 /(1+$ $\left.\left(u_{\mathrm{AMG}-i} i\right)\right)^{2}<0$ by taking derivative, so $g\left(u_{\mathrm{AMG}-i}\right)$ is a decreasing function. Suppose $\min \left(u_{\mathrm{AMG}}\right) \leq u_{\mathrm{AMG}-i} \leq \max$ $\left(u_{\mathrm{AMG}}\right)$ for all $j$, we can get $\left(\left(1-\max \left(u_{\mathrm{AMG}}\right)\right) /(1+\max \right.$ $\left.\left.\left(u_{\mathrm{AMG}}\right)\right)\right) \leq\left(\left(1-\left(u_{\mathrm{AMG}-i}\right)\right) /\left(1+\left(u_{\mathrm{AMG}-i}\right)\right)\right) \leq\left(\left(1-\min \left(u_{\mathrm{AMG}}\right)\right)\right.$ $\left./\left(1+\min \left(u_{\mathrm{AMG}}\right)\right)\right)$; then,

$$
\begin{aligned}
& \left(\frac{1-\max \left(u_{\mathrm{AMG}}\right)}{1+\max \left(u_{\mathrm{AMG}}\right)}\right)^{\left(\left(1+T\left(A_{\mathrm{LD}-i}\right)\right) / \sum_{i=1}^{n}\left(1+T\left(A_{\mathrm{LD}-i}\right)\right)\right)} \leq\left(\frac{1-\left(u_{\mathrm{AMG}-i}\right)}{1+\left(u_{\mathrm{AMG}-i}\right)}\right)^{\left(1+T\left(A_{\mathrm{LD}-i}\right)\right) / \sum_{i=1}^{n}\left(1+T\left(A_{\mathrm{LD}-i}\right)\right)} \\
& \quad \leq\left(\frac{1-\min \left(u_{\mathrm{AMG}}\right)}{1+\min \left(u_{\mathrm{AMG}}\right)}\right)^{\left(1+T\left(A_{\mathrm{LD}-i}\right)\right) / \sum_{i=1}^{n}\left(1+T\left(A_{\mathrm{LD}-i}\right)\right)}, \\
& \prod_{i=1}^{n}\left(\frac{1-\max \left(u_{\mathrm{AMG}}\right)}{1+\max \left(u_{\mathrm{AMG}}\right)}\right)^{\left(1+T\left(A_{\mathrm{LD}-i}\right)\right) / \sum_{i=1}^{n}\left(1+T\left(A_{\mathrm{LD}-i}\right)\right)} \leq \prod_{i=1}^{n}\left(\frac{1-\left(u_{\mathrm{AMG}-i}\right)}{1+\left(u_{\mathrm{AMG}-i}\right)}\right)^{\left(1+T\left(A_{\mathrm{LD}-i}\right)\right) / \sum_{i=1}^{n}\left(1+T\left(A_{\mathrm{LD}-i}\right)\right)} \\
& \quad \leq \prod_{i=1}^{n}\left(\frac{1-\min \left(u_{\mathrm{AMG}}\right)}{1+\min \left(u_{\mathrm{AMG}}\right)}\right)^{\left(1+T\left(A_{\mathrm{LD}-i}\right)\right) / \sum_{i=1}^{n}\left(1+T\left(A_{\mathrm{LD}-i}\right)\right)}, \\
& \left(\frac{1-\max \left(u_{\mathrm{AMG}}\right)}{1+\max \left(u_{\mathrm{AMG}}\right)}\right)^{\sum_{i=1}^{n}\left(1+T\left(A_{\mathrm{LD}-i}\right)\right) / \sum_{i=1}^{n}\left(1+T\left(A_{\mathrm{LD}-i}\right)\right)} \leq\left(\frac{1-\min \left(u_{\mathrm{AMG}}\right)}{1+\min \left(u_{\mathrm{AMG}}\right)}\right)^{\sum_{i=1}^{n}\left(1+T\left(A_{\mathrm{LD}-i}\right)\right) / \sum_{i=1}^{n}\left(1+T\left(A_{\mathrm{LD}-i}\right)\right)} \\
& \quad \leq \prod_{i=1}^{n}\left(\frac{1-\left(u_{\mathrm{AMG}-i}\right)}{1+\left(u_{\mathrm{AMG}-i}\right)}\right)^{\left(1+T\left(A_{\mathrm{LD}-i}\right)\right) / \sum_{i=1}^{n}\left(1+T\left(A_{\mathrm{LD}-i}\right)\right)} \leq
\end{aligned}
$$


i.e.,

$$
\begin{aligned}
& \frac{1-\max \left(u_{\mathrm{AMG}}\right)}{1+\max \left(u_{\mathrm{AMG}}\right)} \leq \prod_{i=1}^{n}\left(\frac{1-\left(u_{\mathrm{AMG}-i}\right)}{1+\left(u_{\mathrm{AMG}-i}\right)}\right)^{\left(1+T\left(A_{\mathrm{LD}-i}\right)\right) / \sum_{i=1}^{n}\left(1+T\left(A_{\mathrm{LD}-i}\right)\right)} \leq \frac{1-\min \left(u_{\mathrm{AMG}}\right)}{1+\min \left(u_{\mathrm{AMG}}\right)}, \\
& \frac{2}{1+\max \left(u_{\mathrm{AMG}}\right)} \leq 1+\prod_{i=1}^{n}\left(\frac{1-\left(u_{\mathrm{AMG}-i}\right)}{1+\left(u_{\mathrm{AMG}-i}\right)}\right)^{\left(1+T\left(A_{\mathrm{LD}-i}\right)\right) / \sum_{i=1}^{n}\left(1+T\left(A_{\mathrm{LD}-i}\right)\right)} \leq \frac{2}{1+\min \left(u_{\mathrm{AMG}}\right)},
\end{aligned}
$$

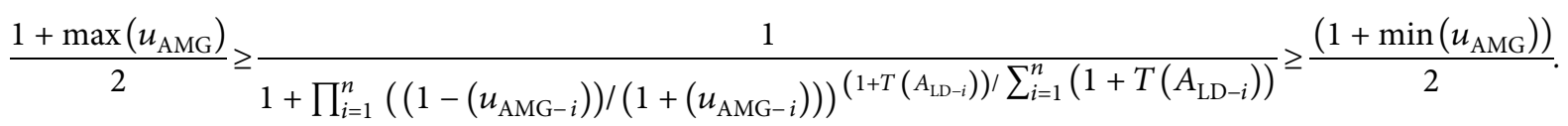

Thus,

$$
\begin{aligned}
1+\max \left(u_{\mathrm{AMG}}\right) & \geq \frac{2}{1+\prod_{i=1}^{n}\left(\left(1-\left(u_{\mathrm{AMG}-i}\right)\right) /\left(1+\left(u_{\mathrm{AMG}-i}\right)\right)\right)\left(1+T\left(A_{\mathrm{LD}-i}\right)\right) / \sum_{i=1}^{n}\left(1+T\left(A_{\mathrm{LD}-i}\right)\right)} \geq 1+\min \left(u_{\mathrm{AMG}}\right), \\
\max \left(u_{\mathrm{AMG}}\right) & \geq \frac{2}{1+\prod_{i=1}^{n}\left(\left(1-\left(u_{\mathrm{AMG}-i}\right)\right) /\left(1+\left(u_{\mathrm{AMG}-i}\right)\right)\right)\left(1+T\left(A_{\mathrm{LD}-i}\right)\right) / \sum_{i=1}^{n}\left(1+T\left(A_{\mathrm{LD}-i}\right)\right)}-1 \geq \min \left(u_{\mathrm{AMG}}\right) .
\end{aligned}
$$

Therefore,

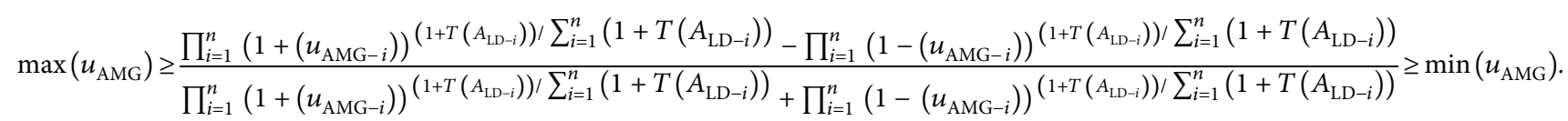

Second, let $f\left(v_{\mathrm{ANG}-i}\right)=\left(2-v_{\mathrm{ANG}-i}\right) / v_{\mathrm{ANG}-i}, v_{\mathrm{ANG}-i} \epsilon$ $[0,1]$, and we can get $f\left(v_{\mathrm{ANG}-i}\right)=-2 /\left(v_{\mathrm{ANG}-i}\right)^{2}<0$ by taking derivative, so $f\left(v_{\mathrm{AMG}-i}\right)$ is a decreasing function.
Suppose $\min \left(v_{\mathrm{AMG}}\right) \leq v_{\mathrm{AMG}-i} \leq \max \left(v_{\mathrm{AMG}}\right)$ for all $i$, we can get $\left(2-\min \left(v_{\mathrm{AMG}}\right)\right) / \min \left(v_{\mathrm{AMG}}\right) \leq\left(2-\left(v_{\mathrm{AMG}-i}\right)\right) /\left(v_{\mathrm{AMG}-i}\right)$ $\leq\left(2-\max \left(v_{\mathrm{AMG}}\right)\right) / \max \left(v_{\mathrm{AMG}}\right)$; then, we have

$$
\begin{aligned}
& \left(\frac{2-\min \left(v_{\mathrm{AMG}}\right)}{\min \left(v_{\mathrm{AMG}}\right)}\right)^{\left(1+T\left(A_{\mathrm{LD}-i}\right)\right) / \sum_{i=1}^{n}\left(1+T\left(A_{\mathrm{LD}-i}\right)\right)} \leq\left(\frac{2-\left(v_{\mathrm{AMG}-i}\right)}{\left(v_{\mathrm{AMG}-i}\right)}\right)^{\left(1+T\left(A_{\mathrm{LD}-i}\right)\right) / \sum_{i=1}^{n}\left(1+T\left(A_{\mathrm{LD}-i}\right)\right)} \\
& \leq\left(\frac{2-\max \left(v_{\mathrm{AMG}}\right)}{\max \left(v_{\mathrm{AMG}}\right)}\right)^{\left(1+T\left(A_{\mathrm{LD}-i}\right)\right) / \sum_{i=1}^{n}\left(1+T\left(A_{\mathrm{LD}-i}\right)\right)}, \\
& \left(\frac{2-\min \left(v_{\mathrm{AMG}}\right)}{\min \left(v_{\mathrm{AMG}}\right)}\right)^{\sum_{i=1}^{n}\left(\left(1+T\left(A_{\mathrm{LD}-i}\right)\right) / \sum_{i=1}^{n}\left(1+T\left(A_{\mathrm{LD}-i}\right)\right)\right)} \\
& \leq \prod_{i=1}^{n}\left(\frac{2-\left(v_{\mathrm{AMG}-i}\right)}{\left(v_{\mathrm{AMG}-i}\right)}\right)^{\left(1+T\left(A_{\mathrm{LD}-i}\right)\right) / \sum_{i=1}^{n}\left(1+T\left(A_{\mathrm{LD}-i}\right)\right)} \leq\left(\frac{2-\max \left(v_{\mathrm{AMG}}\right)}{\max \left(v_{\mathrm{AMG}}\right)}\right)^{\sum_{i=1}^{n}\left(\left(1+T\left(A_{\mathrm{LD}-i}\right)\right) / \sum_{i=1}^{n}\left(1+T\left(A_{\mathrm{LD}-i}\right)\right)\right),} \\
& \frac{2-\min \left(v_{\mathrm{AMG}}\right)}{\min \left(v_{\mathrm{AMG}}\right)} \leq \prod_{i=1}^{n}\left(\frac{2-\left(v_{\mathrm{AMG}-i}\right)}{\left(v_{\mathrm{AMG}-i}\right)}\right)^{\left(1+T\left(A_{\mathrm{LD}-i}\right)\right) / \sum_{i=1}^{n}\left(1+T\left(A_{\mathrm{LD}-i}\right)\right)} \leq \frac{2-\max \left(v_{\mathrm{AMG}}\right)}{\max \left(v_{\mathrm{AMG}}\right)},
\end{aligned}
$$




$$
\begin{aligned}
& \frac{2}{\min \left(v_{\mathrm{AMG}}\right)} \leq \prod_{i=1}^{n}\left(\frac{2-\left(v_{\mathrm{AMG}-i}\right)}{\left(v_{\mathrm{AMG}-i}\right)}\right)^{\left(1+T\left(A_{\mathrm{LD}-i}\right)\right) / \sum_{i=1}^{n}\left(1+T\left(A_{\mathrm{LD}-i}\right)\right)}+1 \leq \frac{2}{\max \left(v_{\mathrm{AMG}}\right)},
\end{aligned}
$$

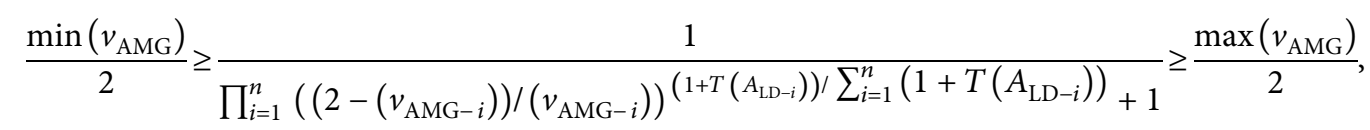

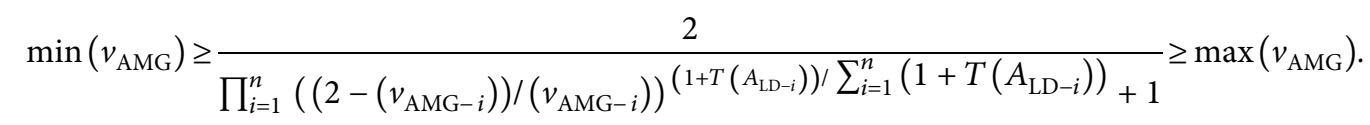

Therefore,

$$
\min \left(v_{\mathrm{AMG}}\right) \geq \frac{2 \prod_{i=1}^{n}\left(v_{\mathrm{AMG}-i}\right)\left(1+T\left(A_{\mathrm{LD}-i}\right)\right) / \sum_{i=1}^{n}\left(1+T\left(A_{\mathrm{LD}-i}\right)\right)}{\prod_{i=1}^{n}\left(2-\left(v_{\mathrm{AMG}-i}\right)\right)^{\left(1+T\left(A_{\mathrm{LD}-i}\right)\right) / \sum_{i=1}^{n}\left(1+T\left(A_{\mathrm{LD}-i}\right)\right)}+\prod_{i=1}^{n}\left(v_{\mathrm{AMG}-i}\right)\left(1+T\left(A_{\mathrm{LD}-i}\right)\right) / \sum_{i=1}^{n}\left(1+T\left(A_{\mathrm{LD}-i}\right)\right)} \geq \max \left(v_{\mathrm{AMG}}\right) .
$$

For $\alpha$ and $\beta$ because $s_{A-\theta_{\min }} \leq s_{A-\theta_{i}} \leq s_{A-\theta_{\max }}$, $s_{A-\tau_{\min }} \leq s_{A-\tau_{i}} \leq s_{A-\tau_{\max }}$ for all $i$, then

$$
\begin{aligned}
& \mathcal{S}\left(A-\theta_{\min }\right){ }^{\left(1+T\left(A_{\mathrm{LD}-i}\right)\right) / \sum_{i=1}^{n}\left(1+T\left(A_{\mathrm{LD}-i}\right)\right)} \leq \mathcal{S}\left(A-\theta_{i}\right)^{\left(1+T\left(A_{\mathrm{LD}-i}\right)\right) / \sum_{i=1}^{n}\left(1+T\left(A_{\mathrm{LD}-i)}\right)\right.} \leq \mathcal{S}\left(A-\theta_{\max }\right)^{\left(1+T\left(A_{\mathrm{LD}-i}\right)\right) / \sum_{i=1}^{n}\left(1+T\left(A_{\mathrm{LD}-i}\right)\right)}, \\
& \left.\mathcal{S}_{\left(A-\tau_{\min }\right)}\right)^{\left(1+T\left(A_{\mathrm{LD}-i}\right)\right) / \sum_{i=1}^{n}\left(1+T\left(A_{\mathrm{LD}-i}\right)\right)} \leq \boldsymbol{S}\left(A-\tau_{i}\right)^{\left(1+T\left(A_{\mathrm{LD}-i}\right)\right) / \sum_{i=1}^{n}\left(1+T\left(A_{\mathrm{LD}-i}\right)\right)} \leq \mathcal{S}\left(A-\tau_{\max }\right)^{\left(1+T\left(A_{\mathrm{LD}-i}\right)\right) / \sum_{i=1}^{n}\left(1+T\left(A_{\mathrm{LD}-i}\right)\right)}, \\
& \mathcal{S} \sum_{i=1}^{n}\left(A-\theta_{\min }\right){ }^{\left(1+T\left(A_{\mathrm{LD}-i}\right)\right) / \sum_{i=1}^{n}\left(1+T\left(A_{\mathrm{LD}-i}\right)\right)} \leq \mathcal{S} \sum_{i=1}^{n}\left(A-\theta_{i}\right)^{\left(1+T\left(A_{\mathrm{LD}-i}\right)\right) /} \sum_{i=1}^{n}\left(1+T\left(A_{\mathrm{LD}-i}\right)\right) \\
& \leq S \sum_{i=1}^{n}\left(A-\theta_{\max }\right)\left(1+T\left(A_{\mathrm{LD}-i}\right)\right) / \sum_{i=1}^{n}\left(1+T\left(A_{\mathrm{LD}-i}\right)\right),
\end{aligned}
$$

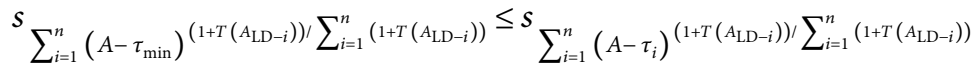

$$
\begin{aligned}
& \leq S \sum_{i=1}^{n}\left(A-\tau_{\max }\right)\left(1+T\left(A_{\mathrm{LD}-i}\right)\right) / \sum_{i=1}^{n}\left(1+T\left(A_{\mathrm{LD}-i}\right)\right),
\end{aligned}
$$

i.e., $\quad s_{A-\theta_{\min }} \leq s \sum_{i=1}^{n}\left(\left(A-\theta_{i}\right)\left(1+T\left(A_{\mathrm{LD}-i}\right)\right) / \sum_{i=1}^{n}\left(1+T\left(A_{\mathrm{LD}-i}\right)\right)\right) \leq s_{A-\theta_{\max }}$, $s_{A-\tau_{\min }} \leq s_{\sum_{i=1}^{n}\left(\left(A-\tau_{i}\right)\left(1+T\left(A_{\mathrm{LD}-i}\right)\right) / \sum_{i=1}^{n}\left(1+T\left(A_{\mathrm{LD}-i}\right)\right)\right)} \leq s_{A-\tau_{\max }}$.

If $\quad$ LDULFPEA $\left(A_{\mathrm{LD}-1}, A_{\mathrm{LD}-2}, \ldots, \quad A_{\mathrm{LD}-n}\right)=A=$ $\left(\left[s_{A-\theta}, s_{A-\tau}\right],\left(u_{\mathrm{AMG}}, v_{\mathrm{ANG}}\right),\left(\alpha_{\mathrm{AMG}}, \beta_{\mathrm{ANG}}\right)\right)$, we know that
$s_{A-\theta_{\min }} \leq s_{A-\theta} \leq s_{A-\theta_{\max }}, \quad s_{A-\tau_{\min }} \leq s_{A-\tau} \leq s_{A-\tau_{\max }}, \quad \min \left(u_{\mathrm{AMG}}\right)$ $\leq u_{\mathrm{AMG}} \leq \max \left(u_{\mathrm{AMG}}\right), \min \left(v_{\mathrm{ANG}}\right) \leq v_{\mathrm{ANG}} \leq \max \left(v_{\mathrm{ANG}}\right)$, $\min \left(\alpha_{\mathrm{AMG}}\right) \leq \alpha_{\mathrm{AMG}} \leq \max \left(\alpha_{\mathrm{AMG}}\right)$, and $\min \left(\beta_{\mathrm{ANG}}\right) \leq \beta_{\mathrm{ANG}}$ $\leq \max \left(\beta_{\mathrm{ANG}}\right)$.

$$
\begin{aligned}
& s_{A-\theta_{\min }}+s_{A-\tau_{\min }} \leq s_{A-\theta}+s_{A-\tau} \leq s_{A-\theta_{\max }}+s_{A-\tau_{\max }} \\
& \min \left(u_{\mathrm{AMG}}\right)-\min \left(v_{\mathrm{ANG}}\right) \leq u_{\mathrm{AMG}}-v_{\mathrm{ANG}} \leq \max \left(u_{\mathrm{AMG}}\right)-\max \left(v_{\mathrm{ANG}}\right), \\
& \min \left(\alpha_{\mathrm{AMG}}\right)-\min \left(\beta_{\mathrm{ANG}}\right) \leq \alpha_{\mathrm{AMG}}-\beta_{\mathrm{ANG}} \leq \max \left(\alpha_{\mathrm{AMG}}\right)-\max \left(\beta_{\mathrm{ANG}}\right) .
\end{aligned}
$$


Therefore, $\min A \leq \operatorname{LDULFPEA}\left(A_{\mathrm{LD}-1}, A_{\mathrm{LD}-2}, \ldots, A_{\mathrm{LD}-n}\right) \leq \max A$.
Theorem 6. For any two LDULNs $A_{L D-i}=\left(\left[s_{A-\theta_{i}}\right.\right.$, $\left.\left.s_{A-\tau_{i}}\right],\left(u_{A M G-i}, v_{A N G-i}\right),\left(\alpha_{A M G-i}, \beta_{A N G-i}\right)\right)$ and $B_{L D-i}=$ $\left(\left[s_{B-\theta_{i}}, s_{B-\tau_{i}}\right],\left(u_{B M G-i}, v_{B N G-i}\right),\left(\alpha_{B M G-i}, \beta_{B N G-i}\right)\right)$ and if $s_{A-\theta_{i}} \leq s_{B-\theta_{i}}, s_{A-\tau_{i}} \leq s_{B-\tau_{i}}, u_{A M G-i} \leq u_{B M G-i}, \quad v_{A N G-i} \leq v_{B N G-i}$, $\alpha_{A M G-i} \leq \alpha_{B M G-i}$ and $\beta_{A N G-i} \leq \beta_{B N G-i}$, for all $i=1,2, \ldots, n$, then

$$
\operatorname{LDULFPEA}\left(A_{\mathrm{LD}-1}, A_{\mathrm{LD}-2}, \ldots, A_{\mathrm{LD}-n}\right) \leq \operatorname{LDULFPEA}\left(B_{\mathrm{LD}-1}, B_{\mathrm{LD}-2}, \ldots, B_{\mathrm{LD}-n}\right) .
$$

Proof. Since $s_{A-\theta_{i}}+s_{A-\tau_{i}} \leq s_{B-\theta_{i}}+s_{B-\tau_{i}}, \quad u_{\mathrm{AMG}-i} \leq u_{\mathrm{BMG}-i}$, $v_{\mathrm{ANG}-i} \leq v_{\mathrm{BNG}-i}, \alpha_{\mathrm{AMG}-i} \leq \alpha_{\mathrm{BMG}-i}$, and $\beta_{\mathrm{ANG}-i} \leq \beta_{\mathrm{BNG}-i}$, for all $i$, we can get

$$
\begin{aligned}
& \sum_{i=1}^{n}\left(\frac{1+T\left(A_{\mathrm{LD}-i}\right)}{\sum_{i=1}^{n}\left(1+T\left(A_{\mathrm{LD}-i}\right)\right)}\left(s_{A-\theta_{i}}+s_{A-\tau_{i}}\right)\right) \leq \sum_{i=1}^{n}\left(\frac{1+T\left(A_{\mathrm{LD}-i}\right)}{\sum_{i=1}^{n}\left(1+T\left(A_{\mathrm{LD}-i}\right)\right)}\left(s_{B-\theta_{i}}+s_{B-\tau_{i}}\right)\right) \\
& { }^{s} \sum_{i=1}^{n}\left(A-\theta_{i}\right)\left(\left(1+T\left(A_{\mathrm{LD}-i}\right)\right) / \sum_{i=1}^{n}\left(1+T\left(A_{\mathrm{LD}-i}\right)\right)\right)+s \\
& { }^{\leq} \sum_{i=1}^{n}\left(A-\tau_{i}\right)\left(\left(1+T\left(A_{\mathrm{LD}-i}\right)\right) / \sum_{i=1}^{n}\left(1+T\left(A_{\mathrm{LD}-i}\right)\right)\right) \\
& \sum_{i=1}^{n}\left(B-\theta_{i}\right)\left(\left(1+T\left(B_{\mathrm{LD}-i}\right)\right) / \sum_{i=1}^{n}\left(1+T\left(B_{\mathrm{LD}-i}\right)\right)\right)^{+s} \sum_{i=1}^{n}\left(B-\tau_{i}\right)\left(\left(1+T\left(B_{\mathrm{LD}-i}\right)\right) / \sum_{i=1}^{n}\left(1+T\left(B_{\mathrm{LD}-i}\right)\right)\right)
\end{aligned}
$$

Since $\quad\left(\left(1-u_{\mathrm{BMG}-i}\right) / 1+\quad u_{\mathrm{BMG}-i}\right) \leq\left(\left(1-u_{\mathrm{AMG}-i}\right) /\right.$ $\left.\left(1+u_{\mathrm{AMG}-i}\right)\right), i=1,2, \ldots, n$, then

$$
\begin{aligned}
& \prod_{i=1}^{n}\left(\frac{1-\left(u_{\mathrm{BMG}-i}\right)}{1+\left(u_{\mathrm{BMG}-i}\right)}\right)^{\left(1+T\left(B_{\mathrm{LD}-i}\right)\right) / \sum_{i=1}^{n}\left(1+T\left(B_{\mathrm{LD}-i}\right)\right)} \leq \prod_{i=1}^{n}\left(\frac{1-\left(u_{\mathrm{AMG}-i}\right)}{1+\left(u_{\mathrm{AMG}-i}\right)}\right)^{\left(1+T\left(A_{\mathrm{LD}-i}\right)\right) / \sum_{i=1}^{n}\left(1+T\left(A_{\mathrm{LD}-i}\right)\right)} \\
& 2 \\
& \overline{1+\prod_{i=1}^{n}\left(\left(1-\left(u_{\mathrm{BMG}-i}\right)\right) /\left(1+\left(u_{\mathrm{BMG}-i}\right)\right)\right)^{\left(1+T\left(B_{\mathrm{LD}-i}\right)\right) / \sum_{i=1}^{n}\left(1+T\left(B_{\mathrm{LD}-i}\right)\right)}} \\
& \geq \frac{2}{1+\prod_{i=1}^{n}\left(\left(1-\left(u_{\mathrm{AMG}-i}\right)\right) /\left(1+\left(u_{\mathrm{AMG}-i}\right)\right)\right)^{\left(1+T\left(A_{\mathrm{LD}-i}\right)\right) / \sum_{i=1}^{n}\left(1+T\left(A_{\mathrm{LD}-i}\right)\right)}} .
\end{aligned}
$$

So, we have

$$
\begin{aligned}
& \frac{\prod_{i=1}^{n}\left(1+\left(u_{\mathrm{AMG}-i}\right)\right)^{\left(1+T\left(A_{\mathrm{LD}-i}\right)\right) / \sum_{i=1}^{n}\left(1+T\left(A_{\mathrm{LD}-i}\right)\right)}-\prod_{i=1}^{n}\left(1-\left(u_{\mathrm{AMG}-i}\right)\right)^{\left(1+T\left(A_{\mathrm{LD}-}\right)\right) / \sum_{i=1}^{n}\left(1+T\left(A_{\mathrm{LD}-i}\right)\right)}}{\prod_{i=1}^{n}\left(1+\left(u_{\mathrm{AMG}-i}\right)\right)^{\left(1+T\left(A_{\mathrm{LD}-i}\right)\right) / \sum_{i=1}^{n}\left(1+T\left(A_{\mathrm{LD}-i}\right)\right)}+\prod_{i=1}^{n}\left(1-\left(u_{\mathrm{AMG}-i}\right)\right)^{\left(1+T\left(A_{\mathrm{LD}-i}\right)\right) / \sum_{i=1}^{n}\left(1+T\left(A_{\mathrm{LD}-i}\right)\right)}} \\
& \geq \frac{\prod_{i=1}^{n}\left(1+\left(u_{\mathrm{BMG}-i}\right)\right)^{\left(1+T\left(B_{\mathrm{LD}-i}\right)\right) / \sum_{i=1}^{n}\left(1+T\left(B_{\mathrm{LD}-i}\right)\right)}-\prod_{i=1}^{n}\left(1-\left(u_{\mathrm{BMG}-i}\right)\right)^{\left(1+T\left(B_{\mathrm{LD}-i}\right)\right) / \sum_{i=1}^{n}\left(1+T\left(B_{\mathrm{LD}-i}\right)\right)}}{\prod_{i=1}^{n}\left(1+\left(u_{\mathrm{BMG}-i}\right)\right)^{\left(1+T\left(B_{\mathrm{LD}-i}\right)\right) / \sum_{i=1}^{n}\left(1+T\left(B_{\mathrm{LD}-i}\right)\right)}+\prod_{i=1}^{n}\left(1-\left(u_{\mathrm{BMG}-i}\right)\right)^{\left(1+T\left(B_{\mathrm{LD}-i}\right)\right) / \sum_{i=1}^{n}\left(1+T\left(B_{\mathrm{LD}-i}\right)\right)}}
\end{aligned}
$$


For $\alpha_{\mathrm{AMG}-i}, \alpha_{\mathrm{BMG}-i}$, we can get

$$
\begin{aligned}
& \frac{\prod_{i=1}^{n}\left(1+\alpha_{\mathrm{AMG}-i}\right)^{\left(1+T\left(A_{\mathrm{LD}-i}\right)\right) / \sum_{i=1}^{n}\left(1+T\left(A_{\mathrm{LD}-i}\right)\right)}-\prod_{i=1}^{n}\left(1-\alpha_{\mathrm{AMG}-i}\right)^{\left(1+T\left(A_{\mathrm{LD}-i}\right)\right) / \sum_{i=1}^{n}\left(\left(1+T\left(A_{\mathrm{LD}-i}\right)\right)\right)}}{\prod_{i=1}^{n}\left(1+\alpha_{\mathrm{AMG}-i}\right)^{\left(1+T\left(A_{\mathrm{LD}-i}\right)\right) / \sum_{i=1}^{n}\left(\left(1+T\left(A_{\mathrm{LD}-i}\right)\right)\right)}+\prod_{i=1}^{n}\left(1-\alpha_{\mathrm{AMG}-i}\right)^{\left(1+T\left(A_{\mathrm{LD}-i}\right)\right) / \sum_{i=1}^{n}\left(\left(1+T\left(A_{\mathrm{LD}-i}\right)\right)\right)}} \\
& \geq \frac{\prod_{i=1}^{n}\left(1+\alpha_{\mathrm{BMG}-i}\right)^{\left(1+T\left(B_{\mathrm{LD}-i}\right)\right) / \sum_{i=1}^{n}\left(1+T\left(B_{\mathrm{LD}-i}\right)\right)}-\prod_{i=1}^{n}\left(1-\alpha_{\mathrm{BMG}-i}\right)^{\left(1+T\left(B_{\mathrm{LD}-i}\right)\right) / \sum_{i=1}^{n}\left(1+T\left(B_{\mathrm{LD}-i}\right)\right)}}{\prod_{i=1}^{n}\left(1+\alpha_{\mathrm{BMG}-i}\right)^{\left(1+T\left(B_{\mathrm{LD}-i}\right)\right) / \sum_{i=1}^{n}\left(1+T\left(B_{\mathrm{LD}-i}\right)\right)}+\prod_{i=1}^{n}\left(1-\alpha_{\mathrm{BMG}-i}\right)^{\left(1+T\left(B_{\mathrm{LD}-i}\right)\right) / \sum_{i=1}^{n}\left(1+T\left(B_{\mathrm{LD}-i}\right)\right)}}
\end{aligned}
$$

Since $\left(\left(2-v_{\mathrm{BNG}-i}\right) / v_{\mathrm{BNG}}-i\right) \geq\left(\left(2-v_{\mathrm{ANG}-i}\right) / v_{\mathrm{ANG}-i}\right), i=$ $1,2, \ldots, n$, then

$$
\begin{aligned}
& \left(\frac{2-\left(v_{\mathrm{BMG}-i}\right)}{\left(v_{\mathrm{BMG}-i}\right)}\right)^{\left(1+T\left(A_{\mathrm{LD}-i}\right)\right) / \sum_{i=1}^{n}\left(1+T\left(A_{\mathrm{LD}-i}\right)\right)} \geq\left(\frac{2-\left(v_{\mathrm{AMG}-i}\right)}{\left(v_{\mathrm{AMG}-i}\right)}\right)^{\left(1+T\left(A_{\mathrm{LD}-i}\right)\right) / \sum_{i=1}^{n}\left(1+T\left(A_{\mathrm{LD}-i}\right)\right)}, \\
& \prod_{i=1}^{n}\left(\frac{2-\left(v_{\mathrm{BMG}-i}\right)}{\left(v_{\mathrm{BMG}-i}\right)}\right)^{\left(1+T\left(B_{\mathrm{LD}-i}\right)\right) / \sum_{i=1}^{n}\left(1+T\left(B_{\mathrm{LD}-i}\right)\right)} \geq \prod_{i=1}^{n}\left(\frac{2-\left(v_{\mathrm{AMG}-i}\right)}{\left(v_{\mathrm{AMG}-i}\right)}\right)^{\left(1+T\left(A_{\mathrm{LD}-i}\right)\right) / \sum_{i=1}^{n}\left(1+T\left(A_{\mathrm{LD}-i}\right)\right)}, \\
& \frac{1}{\prod_{i=1}^{n}\left(\left(\left(2-\left(v_{\mathrm{BMG}-i}\right)\right) /\left(v_{\mathrm{BMG}-i}\right)\right)\left(1+T\left(B_{\mathrm{LD}-i}\right)\right) / \sum_{i=1}^{n}\left(1+T\left(B_{\mathrm{LD}-i}\right)\right)\right)+1} \\
& \leq \frac{1}{\left(\left(2-\left(v_{\mathrm{AMG}-i}\right)\right) /\left(v_{\mathrm{AMG}-i}\right)\right)^{\left(1+T\left(A_{\mathrm{LD}-i}\right)\right) / \sum_{i=1}^{n}\left(1+T\left(A_{\mathrm{LD}-i}\right)\right)}+1}, \\
& \frac{2}{\prod_{i=1}^{n}\left(\left(\left(2-\left(v_{\mathrm{BMG}-i}\right)\right) /\left(v_{\mathrm{BMG}-i}\right)\right)\left(1+T\left(B_{\mathrm{LD}-i}\right)\right) / \sum_{i=1}^{n}\left(1+T\left(B_{\mathrm{LD}-i}\right)\right)\right)+1} \\
& \leq \frac{2}{\left(\left(2-\left(v_{\mathrm{AMG}-i}\right)\right) /\left(v_{\mathrm{AMG}-i}\right)\right)^{\left(1+T\left(A_{\mathrm{LD}-i}\right)\right) / \sum_{i=1}^{n}\left(1+T\left(A_{\mathrm{LD}-i}\right)\right)+1}},
\end{aligned}
$$

so

$$
\begin{aligned}
& 2 \prod_{i=1}^{n}\left(v_{\mathrm{AMG}-i}\right)^{\left(1+T\left(A_{\mathrm{LD}-i}\right)\right) / \sum_{i=1}^{n}\left(1+T\left(A_{\mathrm{LD}-i}\right)\right)}
\end{aligned}
$$

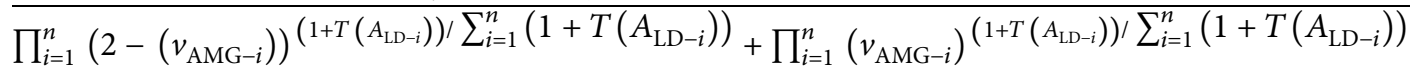

$$
\begin{aligned}
& \leq \frac{2 \prod_{i=1}^{n}\left(v_{\mathrm{BMG}-i}\right)^{\left(1+T\left(B_{\mathrm{LD}-i}\right)\right) / \sum_{i=1}^{n}\left(1+T\left(B_{\mathrm{LD}-i}\right)\right)}}{\prod_{i=1}^{n}\left(2-\left(v_{\mathrm{BMG}-i}\right)\right)^{\left(1+T\left(B_{\mathrm{LD}-i}\right)\right) / \sum_{i=1}^{n}\left(1+T\left(B_{\mathrm{LD}-i}\right)\right)}+\prod_{i=1}^{n}\left(v_{\mathrm{BMG}-i}\right)^{\left(1+T\left(B_{\mathrm{LD}-i}\right)\right) / \sum_{i=1}^{n}\left(1+T\left(B_{\mathrm{LD}-i}\right)\right)} .}
\end{aligned}
$$


For $\beta_{\mathrm{ANG}-i}, \beta_{\mathrm{BNG}-i}$, we can get

$$
\begin{aligned}
& 2 \prod_{i=1}^{n}\left(\beta_{\mathrm{AMG}-i}\right)^{\left(1+T\left(A_{\mathrm{LD}-i}\right)\right) / \sum_{i=1}^{n}\left(1+T\left(A_{\mathrm{LD}-i}\right)\right)}
\end{aligned}
$$

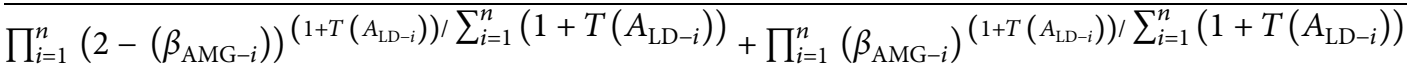

$$
\begin{aligned}
& \leq \frac{2 \prod_{i=1}^{n}\left(\beta_{\mathrm{BNG}-i}\right)^{\left(1+T\left(B_{\mathrm{LD}-i}\right)\right) / \sum_{i=1}^{n}\left(1+T\left(B_{\mathrm{LD}-i}\right)\right)}}{\prod_{i=1}^{n}\left(2-\left(\beta_{\mathrm{BNG}-i}\right)\right)^{\left(1+T\left(B_{\mathrm{LD}-i}\right)\right) / \sum_{i=1}^{n}\left(1+T\left(B_{\mathrm{LD}-i}\right)\right)}+\prod_{i=1}^{n}\left(\beta_{\mathrm{BNG}-i}\right)^{\left(1+T\left(B_{\mathrm{LD}-i}\right)\right) / \sum_{i=1}^{n}\left(1+T\left(B_{\mathrm{LD}-i}\right)\right)} .}
\end{aligned}
$$

Thus, we got that

$\operatorname{LDULFPEA}\left(A_{\mathrm{LD}-1}, A_{\mathrm{LD}-2}, \ldots, A_{\mathrm{LD}-n}\right) \leq \operatorname{LDULFPEA}\left(A_{\mathrm{LD}-1}, A_{\mathrm{LD}-2}, \ldots, A_{\mathrm{LD}-n}\right)$.

Definition 15. For any LDULNs $A_{\mathrm{LD}-i}=\left(\left[s_{A-\theta_{i}}, s_{A-}\right.\right.$ $\left.\left.\tau_{i}\right],\left(u_{\mathrm{AMG}-i}, v_{\mathrm{ANG}-i}\right),\left(\alpha_{\mathrm{AMG}-i}, \beta_{\mathrm{ANG}-i}\right)\right), i=1,2, \ldots, n$, the LDULFPEWA operator is elaborated by

$\operatorname{LDULFPEWA}\left(A_{\mathrm{LD}-1}, A_{\mathrm{LD}-2}, \ldots, A_{\mathrm{LD}-n}\right)=\frac{\otimes_{\varepsilon i=1}^{n} w_{i}\left(1+T\left(A_{\mathrm{LD}-i}\right)\right) * A_{\mathrm{LD}-i}}{\sum_{i=1}^{n} w_{i}\left(1+T\left(A_{\mathrm{LD}-i}\right)\right)}=\otimes_{\varepsilon i=1}^{n}\left(\frac{w_{i}\left(1+T\left(A_{\mathrm{LD}-i}\right)\right) * A_{\mathrm{LD}-i}}{\sum_{i=1}^{n} w_{i}\left(1+T\left(A_{\mathrm{LD}-i}\right)\right)}\right)$,

where $\quad T\left(A_{\mathrm{LD}-i}\right)=\sum_{\substack{j=1 \\ j \neq i}}^{n} \sup \left(A_{\mathrm{LD}-i}, A_{\mathrm{LD}-j}\right), \quad \sup \left(A_{\mathrm{LD}-i}\right.$, $\left.A_{\mathrm{LD}-j}\right)$ is the support for $A_{\mathrm{LD}-i}$ from $A_{\mathrm{LD}-j}$, and $w=\left(w_{1}, w_{2}, \ldots, w_{n}\right)^{T}$ is the weighting vector of the Theorem 7. For any LDULFNs $A_{L D-i}=\left(\left[s_{A-\theta_{i}}, s_{A-\tau_{i}}\right]\right.$, $\left.\left(u_{A M G-i}, v_{A N G-i}\right),\left(\alpha_{A M G-i}, \beta_{A N G-i}\right)\right), i=1,2, \ldots, n$, the result aggregated from Definition 15 is still an LDULFN:

$\left(A_{\mathrm{LD}-1}, A_{\mathrm{LD}-2}, \ldots, A_{\mathrm{LD}-n}\right)$, such that $w_{i} \in[0,1], \sum_{i=1}^{n} w_{i}=1$.

$$
\begin{aligned}
& \operatorname{LDULFPEA}\left(A_{\mathrm{LD}-1}, A_{\mathrm{LD}-2}, \ldots, A_{\mathrm{LD}-D}\right)=\otimes_{\varepsilon}{ }_{i=1}^{n}\left(\frac{w_{i}\left(1+T\left(A_{\mathrm{LD}-i}\right) * A_{\mathrm{LD}-i}\right)}{\sum_{i=1}^{n} w_{i}\left(1+T\left(A_{\mathrm{LD}-i}\right)\right)}\right) \\
& =\left(\left[S \sum_{i=1}^{n}\left(A-\theta_{i} w_{i}\left(1+T\left(A_{\mathrm{LD}-i}\right)\right)\right) / \sum_{i=1}^{n} w_{i}\left(1+T\left(A_{\mathrm{LD}-i}\right)\right), S_{\left.\sum_{i=1}^{n}\left(A-\tau_{i} w_{i}\left(\theta_{i}\left(1+T\left(A_{\mathrm{LD}-i}\right)\right)\right)\right) / \sum_{i=1}^{n} w_{i}\left(1+T\left(A_{\mathrm{LD}-i}\right)\right)\right]}\right]\right.
\end{aligned}
$$

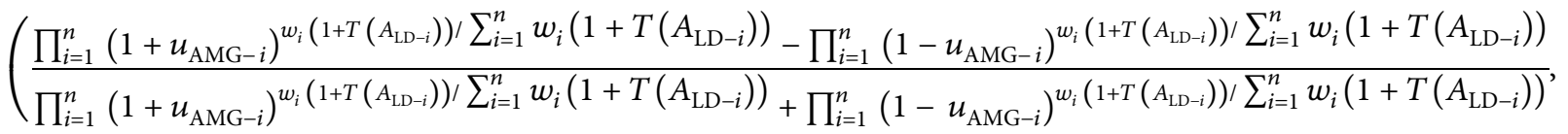

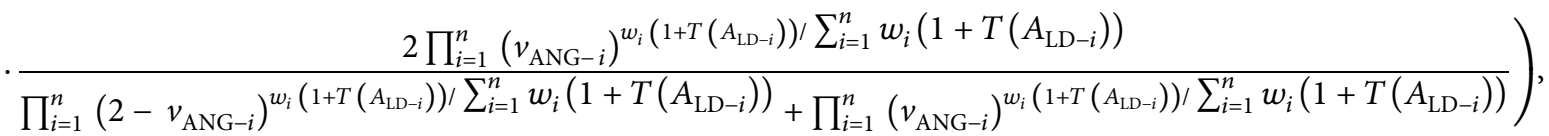

$$
\begin{aligned}
& \left(\frac{\prod_{i=1}^{n}\left(1+\alpha_{\mathrm{AMG}-i}\right)^{w_{i}\left(1+T\left(A_{\mathrm{LD}-i}\right)\right) / \sum_{i=1}^{n} w_{i}\left(1+T\left(A_{\mathrm{LD}-i}\right)\right)}-\prod_{i=1}^{n}\left(1-\alpha_{\mathrm{AMG}-i}\right)^{w_{i}\left(1+T\left(A_{\mathrm{LD}-i}\right)\right) / \sum_{i=1}^{n} w_{i}\left(1+T\left(A_{\mathrm{LD}-i}\right)\right)}}{\prod_{i=1}^{n}\left(1+\alpha_{\mathrm{AMG}-i}\right)^{w_{i}}\left(1+T\left(A_{\mathrm{LD}-i}\right)\right) / \sum_{i=1}^{n} w_{i}\left(1+T\left(A_{\mathrm{LD}-i}\right)\right)+\prod_{i=1}^{n}\left(1-\alpha_{\mathrm{AMG}-i}\right)^{w_{i}}\left(1+T\left(A_{\mathrm{LD}-i}\right)\right) / \sum_{i=1}^{n} w_{i}\left(1+T\left(A_{\mathrm{LD}-i}\right)\right)},\right.
\end{aligned}
$$

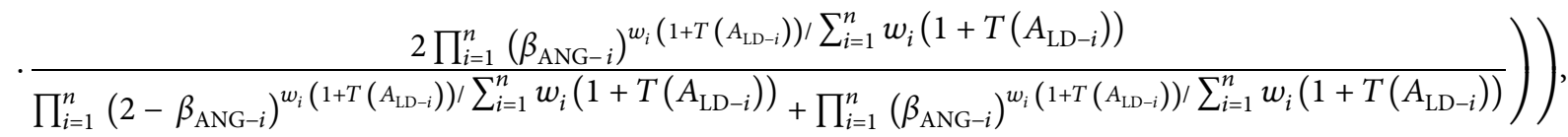


where $T\left(A_{L D-i}\right)=\sum_{\substack{j=1 \\ j \neq i}}^{n} \sup \left(A_{L D-i}, A_{L D-j}\right), \quad \sup \left(A_{L D-i}\right.$, $\left.A_{L D-j}\right)$ is the support for $A_{L D-i}$ from $A_{L D-j}$, and $w=\left(w_{1}\right.$, $\left.w_{2}, \ldots, w_{n}\right)^{T}$ is the weighting vector of the $\left(A_{L D-1}, A_{L D-2}, \ldots, A_{L D-n}\right)$, such that $w_{i} \in[0,1], \sum_{i=1}^{n} w_{i}=1$.

Proof. Trivial.

Theorem 8. For any LDULNs $A_{L D-j}=A, j=1,2, \ldots, n$ and $A=\left(\left[s_{A-\theta}, s_{A-\tau}\right],\left(u_{A M G}, v_{A N G}\right),\left(\alpha_{A M G}, \beta_{A N G}\right)\right)$, then

$$
\operatorname{LDULFPEWA}\left(A_{\mathrm{LD}-1}, A_{\mathrm{LD}-2}, \ldots, A_{\mathrm{LD}-n}\right)=A \text {. }
$$

Proof. Trivial.

Theorem 9. For any LDULNs $A_{L D-i}=\left(\left[s_{A-\theta_{i}}, s_{A-}\right.\right.$ $\left.\left.\tau_{i}\right],\left(u_{A M G-i}, v_{A N G-i}\right),\left(\alpha_{A M G-i}, \beta_{A N G-i}\right)\right), i=1,2, \ldots, n, \quad$ if LDULFPEWA operator lies between the max and min operators,

$$
\begin{aligned}
& \min \left(A_{\mathrm{LD}-1}, A_{\mathrm{LD}-2}, \ldots, A_{\mathrm{LD}-n}\right)=\min A, \\
& \max \left(A_{\mathrm{LD}-1}, A_{\mathrm{LD}-2}, \ldots, A_{\mathrm{LD}-n}\right)=\max A
\end{aligned}
$$

then,

$\min A \leq \operatorname{LDULFPEWA}\left(A_{\mathrm{LD}-1}, A_{\mathrm{LD}-2}, \ldots, A_{\mathrm{LD}-n}\right) \leq \max A$.

Proof. Trivial.

Definition 16. For any LDULNs $A_{\mathrm{LD}-i}=\left(\left[s_{A-\theta_{i}}, s_{A-\tau_{i}}\right]\right.$, $\left.\left(u_{\mathrm{AMG}-i}, v_{\mathrm{ANG}-i}\right),\left(\alpha_{\mathrm{AMG}-i}, \beta_{\mathrm{ANG}-i}\right)\right), i=1,2, \ldots, n$, the LDULFPEA operator is elaborated by

$$
\begin{aligned}
& \operatorname{LDULFPEG}\left(A_{\mathrm{LD}-1}, A_{\mathrm{LD}-2}, \ldots, A_{\mathrm{LD}-n}\right) \\
& =\otimes_{\varepsilon}{ }_{i=1}^{n}\left(A_{\mathrm{LD}-i}\right)^{1+T\left(A_{\mathrm{LD}-i}\right) / \sum_{i=1}^{n}\left(1+T\left(A_{\mathrm{LD}-i}\right)\right),}
\end{aligned}
$$

where $\quad T\left(A_{\mathrm{LD}-i}\right)=\sum_{j=1}^{n} \sup \left(A_{\mathrm{LD}-i}, A_{\mathrm{LD}-j}\right), \quad$ and $j \neq i$

$\sup \left(A_{\mathrm{LD}-i}, A_{\mathrm{LD}-j}\right)$ is the support for $A_{\mathrm{LD}-i}$ from $A_{\mathrm{LD}-j}$.

Theorem 10. For any LDULFNs $A_{L D-i}=\left(\left[s_{A-\theta_{i}}, s_{A-\tau_{i}}\right]\right.$, $\left.\left(u_{A M G-i}, v_{A N G-i}\right),\left(\alpha_{A M G-i}, \beta_{A N G-i}\right)\right), i=1,2, \ldots, n$, the result aggregated from Definition 16 is still an LDULFN:

$$
\begin{aligned}
& \operatorname{LDULFPEA}\left(A_{\mathrm{LD}-1}, A_{\mathrm{LD}-2}, \ldots, A_{\mathrm{LD}-n}\right)=\left(\left[S_{\prod_{i=1}^{n} A=\theta_{i}^{c_{i}}}, S_{\prod_{i=1}^{n} A=\tau_{i}^{c_{i}}}\right],\right. \\
& \left(\frac{2 \prod_{i=1}^{n}\left(u_{\mathrm{ANG}-i}\right)^{c_{i}}}{\prod_{i=1}^{n}\left(2-u_{\mathrm{ANG}-i}\right)^{c_{i}}+\prod_{i=1}^{n}\left(u_{\mathrm{ANG}-i}\right)^{c_{i}}}, \frac{\prod_{i=1}^{n}\left(1+v_{\mathrm{AMG}-i}\right)^{c_{i}}-\prod_{i=1}^{n}\left(1-v_{\mathrm{AMG}-i}\right)^{c_{i}}}{\prod_{i=1}^{n}\left(1+v_{\mathrm{AMG}-i}\right)^{c_{i}}+\prod_{i=1}^{n}\left(1-v_{\mathrm{AMG}-i}\right)^{c_{i}}}\right), \\
& \left.\left(\frac{2 \prod_{i=1}^{n}\left(\alpha_{\mathrm{AMG}-i}\right)^{c_{i}}}{\prod_{i=1}^{n}\left(2-\alpha_{\mathrm{AMG}-i}\right)^{c_{i}}+\prod_{i=1}^{n}\left(\alpha_{\mathrm{AMG}-i}\right)^{c_{i_{i}}}}, \frac{\prod_{i=1}^{n}\left(1+\beta_{\mathrm{AMG}-i}\right)^{c_{i}}-\prod_{i=1}^{n}\left(1-\beta_{\mathrm{AMG}-i}\right)^{c_{i}}}{\prod_{i=1}^{n}\left(2-\beta_{\mathrm{AMG}-i}\right)^{c_{i}}+\prod_{i=1}^{n}\left(1-\beta_{\mathrm{AMG}-i}\right)^{c_{i}}}\right)\right),
\end{aligned}
$$

where $\quad c_{i}=\left(1+T\left(A_{L D-i}\right)\right) / \sum_{i=1}^{n}\left(1+T\left(A_{L D-i}\right)\right)(i=1,2$, $\ldots, n), \quad T\left(A_{L D-i}\right)=\sum_{\substack{j=1 \\ j \neq i}}^{n} \sup \left(A_{L D-i}, A_{L D-j}\right), \quad$ and $\sup \left(A_{L D-i}, A_{L D-j}\right)$ is the support for $A_{L D-i}$ from $A_{L D-j}$.

Proof. Trivial.

$$
\begin{aligned}
& \min \left(A_{\mathrm{LD}-1}, A_{\mathrm{LD}-2}, \ldots, A_{\mathrm{LD}-n}\right)=\min A, \\
& \max \left(A_{\mathrm{LD}-1}, A_{\mathrm{LD}-2}, \ldots, A_{\mathrm{LD}-n}\right)=\max A
\end{aligned}
$$

then,

$$
\min A \leq \operatorname{LDULFPEG}\left(A_{\mathrm{LD}-1}, A_{\mathrm{LD}-2}, \ldots, A_{\mathrm{LD}-n}\right) \leq \max A .
$$

Theorem 11. For any LDULNs $A_{L D-j}=A, j=1,2, \ldots, n$ and $A=\left(\left[s_{A-\theta}, s_{A-\tau}\right],\left(u_{A M G}, v_{A N G}\right),\left(\alpha_{A M G}, \beta_{A N G}\right)\right)$, then

$$
\operatorname{LDULFPEG}\left(A_{\mathrm{LD}-1}, A_{\mathrm{LD}-2}, \ldots, A_{\mathrm{LD}-n}\right)=A \text {. }
$$

Proof. Trivial.

Theorem 12. For any LDULNs $A_{L D-i}=\left(\left[s_{A-\theta_{i}}, s_{A-\tau_{i}}\right]\right.$, $\left.\left(u_{A M G-i}, v_{A N G-i}\right),\left(\alpha_{A M G-i}, \beta_{A N G-i}\right)\right), i=1,2, \ldots, n, \quad$ if LDULFPEG operator lies between the max and min operators,
Proof. Trivial.

Theorem 13. For any two LDULNs $A_{L D-i}=$ $\left(\left[s_{A-\theta_{i}}, s_{A-\tau_{i}}\right],\left(u_{A M G-i}, v_{A N G-i}\right),\left(\alpha_{A M G-i}, \beta_{A N G-i}\right)\right)$ and $B_{L D-i}=\left(\left[s_{B-\theta_{i}}, s_{B-\tau_{i}}\right],\left(u_{B M G-i}, v_{B N G-i}\right),\left(\alpha_{B M G-i}, \beta_{B N G-i}\right)\right)$ and if $\quad s_{A-\theta_{i}} \leq s_{B-\theta_{i}}, s_{A-\tau_{i}} \leq s_{B-} \quad \tau_{i}, u_{A M G-i} \leq u_{B M G-i}, v_{A N G-i} \leq$ $v_{B N G-i}, \alpha_{A M G-i} \leq \alpha_{B M G-i}$ and $\beta_{A N G-i} \leq \beta_{B N G-i}$, for all $i=1,2, \ldots, n$, then 
Proof. Trivial.

Definition 17. For any LDULNs $A_{\mathrm{LD}-i}=\left(\left[s_{A-\theta_{i}}, s_{A-\tau_{i}}\right]\right.$, $\left.\left(u_{\mathrm{AMG}-i}, v_{\mathrm{ANG}-i}\right),\left(\alpha_{\mathrm{AMG}-i}, \beta_{\mathrm{ANG}-i}\right)\right), i=1,2, \ldots, n$, the LDULFPEWG operator is elaborated by

$$
\operatorname{LDULFPEWG}\left(A_{\mathrm{LD}-1}, A_{\mathrm{LD}-2}, \ldots, A_{\mathrm{LD}-n}\right)=\otimes_{\varepsilon}{ }_{i=1}^{n}\left(A_{\mathrm{LD}-i}\right)^{w_{i}\left(1+T\left(A_{\mathrm{LD}-i}\right)\right) / \sum_{i=1}^{n} w_{i}\left(1+T\left(A_{\mathrm{LD}-i}\right)\right)}
$$

where $\quad T\left(A_{\mathrm{LD}-i}\right)=\sum_{\substack{j=1 \\ j \neq i}}^{n} \sup \left(A_{\mathrm{LD}-i}, A_{\mathrm{LD}-j}\right), \quad \sup \left(A_{\mathrm{LD}-i}\right.$, $\left.A_{\mathrm{LD}-j}\right)$ is the support for $A_{\mathrm{LD}-i}$ from $A_{\mathrm{LD}-j}$, and $w=\left(w_{1}, w_{2}, \ldots, w_{n}\right)^{T}$ is the weighting vector of the $\left(A_{\mathrm{LD}-1}, A_{\mathrm{LD}-2}, \ldots, A_{\mathrm{LD}-n}\right)$, such that $w_{i} \in[0,1], \sum_{i=1}^{n} w_{i}=1$.
Theorem 14. For any LDULFNs $A_{L D-i}=\left(\left[s_{A-\theta_{i}}, s_{A-\tau_{i}}\right]\right.$, $\left.\left(u_{A M G-i}, v_{A N G-i}\right),\left(\alpha_{A M G-i}, \beta_{A N G-i}\right)\right), i=1,2, \ldots, n$, then the result aggregated from Definition 17 is still an LDULFN:

$$
\begin{aligned}
& \operatorname{LDULFPEA}\left(A_{\mathrm{LD}-1}, A_{\mathrm{LD}-2}, \ldots, A_{\mathrm{LD}-n}\right)=\otimes_{\varepsilon}{ }_{i=1}^{n}\left(A_{\mathrm{LD}-i}\right)^{w_{i}}\left(1+T\left(A_{\mathrm{LD}-i}\right)\right) / \sum_{i=1}^{n} w_{i}\left(1+T\left(A_{\mathrm{LD}-i}\right)\right)=\left(\left[S_{\prod_{i=1}^{n}} A_{=\theta_{i} b_{i}}, S_{\prod_{i=1}^{n} A=\tau_{i}^{b_{i}}}\right],\right. \\
& \left(\frac{2 \prod_{i=1}^{n}\left(u_{\mathrm{ANG}-i}\right)^{b_{i}}}{\prod_{i=1}^{n}\left(2-u_{\mathrm{ANG}-i}\right)^{b_{i}}+\prod_{i=1}^{n}\left(u_{\mathrm{ANG}-i}\right)^{b_{i}}}, \frac{\prod_{i=1}^{n}\left(1+v_{\mathrm{AMG}-i}\right)^{b_{i}}-\prod_{i=1}^{n}\left(1-v_{\mathrm{AMG}-i}\right)^{b_{i}}}{\prod_{i=1}^{n}\left(1+v_{\mathrm{AMG}-i}\right)^{b_{i}}+\prod_{i=1}^{n}\left(1-v_{\mathrm{AMG}-i}\right)^{b_{i}}}\right), \\
& \left.\left(\frac{2 \prod_{i=1}^{n}\left(\alpha_{\mathrm{AMG}-i}\right)^{b_{i}}}{\prod_{i=1}^{n}\left(2-\alpha_{\mathrm{AMG}-i}\right)^{b_{i}}+\prod_{i=1}^{n}\left(\alpha_{\mathrm{AMG}-i}\right)^{b_{i}}}, \frac{\prod_{i=1}^{n}\left(1+\beta_{\mathrm{AMG}-i}\right)^{b_{i}}-\prod_{i=1}^{n}\left(1-\beta_{\mathrm{AMG}-i}\right)^{b_{i}}}{\prod_{i=1}^{n}\left(2-\beta_{\mathrm{AMG}-i}\right)^{b_{i}}+\prod_{i=1}^{n}\left(1-\beta_{\mathrm{AMG}-i}\right)^{b_{i}}}\right)\right)
\end{aligned}
$$

where $b_{i}=\left(w_{i}\left(1+T\left(A_{L D-i}\right)\right) / \sum_{i=1}^{n} w_{i}\left(1+T\left(A_{L D-i}\right)\right)\right) \quad(i=$ $1,2, \ldots, n), \quad T\left(A_{L D-i}\right)=\sum_{\substack{j=1 \\ j \neq i}}^{n} \sup \left(A_{L D-i}, A_{L D-j}\right), \quad$ and $\sup \left(A_{L D-i}, A_{L D-j}\right)$ is the support for $A_{L D-i}$ from $A_{L D-j} . w=$ $\left(w_{1}, w_{2}, \ldots, w_{n}\right)^{T}$ is the weighting vector of $\left(A_{L D-1}, A_{L D-2}, \ldots, A_{L D-n}\right)$, such that $w_{i} \in[0,1], \sum_{i=1}^{n} w_{i}=1$.

Proof. Trivial.

Theorem 15. For any LDULNs $A_{L D-j}=A, j=1,2, \ldots, n$ and $A=\left(\left[s_{A-\theta}, s_{A-\tau}\right],\left(u_{A M G}, v_{A N G}\right),\left(\alpha_{A M G}, \beta_{A N G}\right)\right)$,

$$
\operatorname{LDULFPEWG}\left(A_{\mathrm{LD}-1}, A_{\mathrm{LD}-2}, \ldots, A_{\mathrm{LD}-n}\right)=A \text {. }
$$

Proof. Trivial.

Theorem 16. For any LDULNs $A_{L D-i}=\left(\left[s_{A-\theta_{i}}, s_{A-\tau_{i}}\right]\right.$, $\left.\left(u_{A M G-i}, v_{A N G-i}\right),\left(\alpha_{A M G-i}, \beta_{A N G-i}\right)\right), i=1,2, \ldots, n, \quad$ if LDULFPEWG operator lies between the max and min operators,

$$
\begin{aligned}
& \min \left(A_{\mathrm{LD}-1}, A_{\mathrm{LD}-2}, \ldots, A_{\mathrm{LD}-n}\right)=\min A, \\
& \max \left(A_{\mathrm{LD}-1}, A_{\mathrm{LD}-2}, \ldots, A_{\mathrm{LD}-n}\right)=\max A
\end{aligned}
$$

then,

$\min A \leq \mathrm{LDULFPEWG}\left(A_{\mathrm{LD}-1}, A_{\mathrm{LD}-2}, \ldots, A_{\mathrm{LD}-n}\right) \leq \max A$
Proof. Trivial.

\section{MADM Technique for LDULSs Using PEAOs}

In this part, we promote the examination of PEAOs of LDULNs in the MADM problem. We promote powered Einstein aggregation operators of LDULNs in the MADM problem. The impact of $\mathfrak{R}$ on the ranking results is investigated thoroughly.

MADM is a way of choosing the best alternative because of some finite attributes using the PE operators of LDULSs. The most favorable thing here is that the information is based on LDULNs which discuss the membership grades and nonmembership grades of the information. Let the collection of alternatives be $A_{k}$ ( $k$ is finite) and attributes be $G_{j}$ ( $j$ is finite), which forms a decision matrix denoted by $D_{k \times j}=(T)_{k \times j}$ $=\left(\left[s_{A-\theta_{k j}}, s_{A-\tau_{k j}}\right],\left(u_{\mathrm{AMG}-k j}, v_{\mathrm{ANG}-k j}\right),\left(\alpha_{\mathrm{AMG}-k j}, \beta_{\mathrm{ANG}-k j}\right)\right)$, where the terms in triplet denote the membership grades, abstinence, and nonmembership grades of the information, where $\omega=\left(\omega_{1}, \omega_{2}, \ldots, \omega_{\mathfrak{r}}\right)^{T}$ is the weight vector of $\left(A_{\mathrm{LD}-1}\right.$, $\left.A_{\mathrm{LD}-2}, \ldots, A_{\mathrm{LD}-\mathfrak{r}}\right), \omega_{j} \in[0,1], \sum_{j=1}^{\mathfrak{r}} \omega_{j}=1$. A brief algorithm of the MADM process is illustrated in the following section.

6.1. Algorithm. The algorithm of MADM based on LDUL information and using LDULPEA, LDULPEWA, LDULPEG, and LDULPEWG operators is proposed as follows:

Step 1: in this step, we collect the information about alternatives given by the decision-makers. The decision-makers gave the opinion about alternates in the 


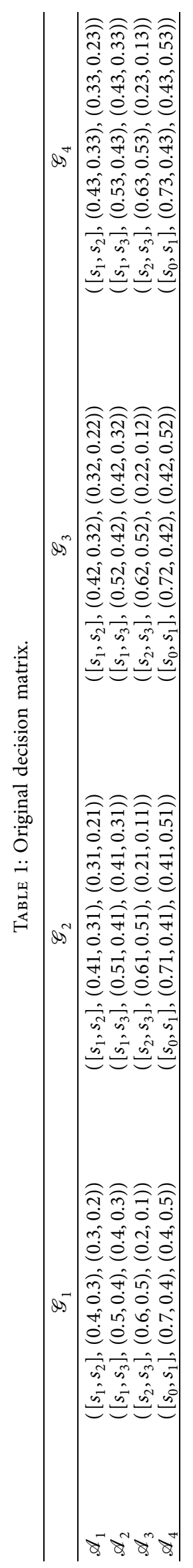




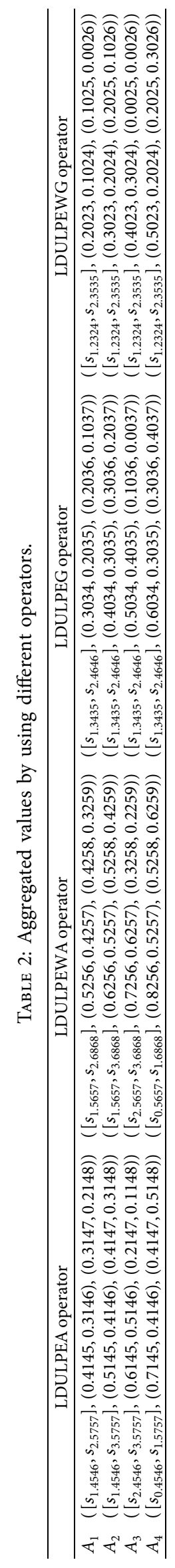


TABLE 3: Score values of the aggregated operators.

\begin{tabular}{lcccc}
\hline Scores & LDULPEA operator & LDULPEWA operator & LDULPEG operator & LDULPEWG operator \\
\hline$A_{1}$ & 0.8209 & 0.9842 & 0.6698 & 0.5401 \\
$A_{2}$ & 0.9216 & 1.0905 & 0.7651 & 0.6298 \\
$A_{3}$ & 0.7201 & 0.8779 & 0.5746 & 0.4953 \\
$A_{4}$ & 1.1231 & 1.3032 & 0.9554 & 0.8091 \\
\hline
\end{tabular}

TABLE 4: Ranking values of the information in Table 3.

\begin{tabular}{lr}
\hline & Ranking analysis \\
\hline LDULPEWA operator & $A_{4}>A_{2}>A_{1}>A_{3}$ \\
LDULPEWG operator & $A_{4}>A_{2}>A_{1}>A_{3}$ \\
LDULPEA operator & $A_{4}>A_{2}>A_{1}>A_{3}$ \\
LDULPEG operator & $A_{4}>A_{2}>A_{1}>A_{3}$ \\
\hline
\end{tabular}

TABLE 5: Comparative analysis of the elaborated operators and existing operators.

\begin{tabular}{lcc}
\hline Method & Score values & Ranking values \\
\hline Riaz and Hashmi [39] & Cannot be calculated & Cannot be calculated \\
Riaz and Hashmi [40] & Cannot be calculated & Cannot be calculated \\
Wang et al. [42] & Cannot be calculated & Cannot be calculated \\
LDULPEWA operator & $0.9842,1.0905,0.8779,1.3032$ & $A_{4}>A_{2}>A_{1}>A_{3}$ \\
LDULPEWG operator & $0.5401,0.6298,0.4953,0.8091$ & $A_{4}>A_{2}>A_{1}>A_{3}$ \\
LDULPEA operator & $0.8209,0.9216,0.7201,1.1231$ & $A_{4}>A_{2}>A_{1}>A_{3}$ \\
LDULPEG operator & $0.6698,0.7651,0.5746,0.9554$ & $A_{4}>A_{2}>A_{1}>A_{3}$ \\
\hline
\end{tabular}

form of LDULNs which leads to the formation of the decision matrix.

Step 2: if there exist attributes of cost type, we normalize the decision matrix by taking the complement of each triplet in the matrix. If not, we use the following LDULPEA, LDULPEWA, LDULPEG, and LDULPEWG operators to aggregate the data given in the decision matrix.

Step 3: in this step, we compute the scores of the aggregated information using Definition 3

Step 4: this step is based on the ranking of the alternatives

An illustrative example to see the viability of the proposed algorithm.

6.2. Illustrated Example. An example of technology commercialization is adapted from [30] where the selection of the most favorable software enterprise among a list of enterprises is carried out. Let us consider four software enterprises denoted by $A_{1 \leq k \leq 4}$, and the attributes are denoted by $G_{1 \leq j \leq 4}$, where $G_{1}$ represents the advancement of the technology, $G_{2}$ represents the market potential, $G_{3}$ represents the human resources and financial development, and $G_{4}$ represents the creating of employment and development of technology. The weight vector chosen in this case is $\omega=(0.4,0.3,0.2,0.1)^{T}$. The selection of the weight vector is up to the decision-makers, and the information about the alternatives in terms of LDULNs is given in Table 1.
Step 1: in this step, the information given in Table 1 is aggregated using LDULPEA, LDULPEWA, LDULPEG, and LDULPEWG operators. The results of the aggregation are given in Table 2 .

Step 2: in this step, the aggregated information obtained in Table 3 is targeted, and their scores are computed using Definition 3.

Step 3: based on the scores obtained in Table 4, the alternatives are ranked, and the ranking results are given in Table 5.

As shown above, we obtained the same ranking results, which are discussed in the form of Table 4 . The best option is $A_{4}$. The results portrayed in Table 3 are further described geometrically in Figure 2.

6.3. Comparative Analysis. Based on the investigated LDULPEA, LDULPEWA, LDULPEG, and LDULPEWG operators, we determined the reliability and consistency of the developed operators with the help of comparative analysis by using the information of Table 1 shown in 6.2. The information related to existing theories is as follows: Riaz and Hashmi [39] elaborated the aggregation operators for LDFS and their applications, Riaz and Hashmi [40] proposed some operators based on linear Diophantine sets, and Wang et al. [42] proposed interaction Hamacher operators for Pythagorean fuzzy sets. The reasonable analysis of the investigated operators and remaining operators are discussed in Table 5 by using the information of Table 1, by using the values of parameters $\mathfrak{f}=\mathfrak{h}=1, \mathfrak{x}=3$. 


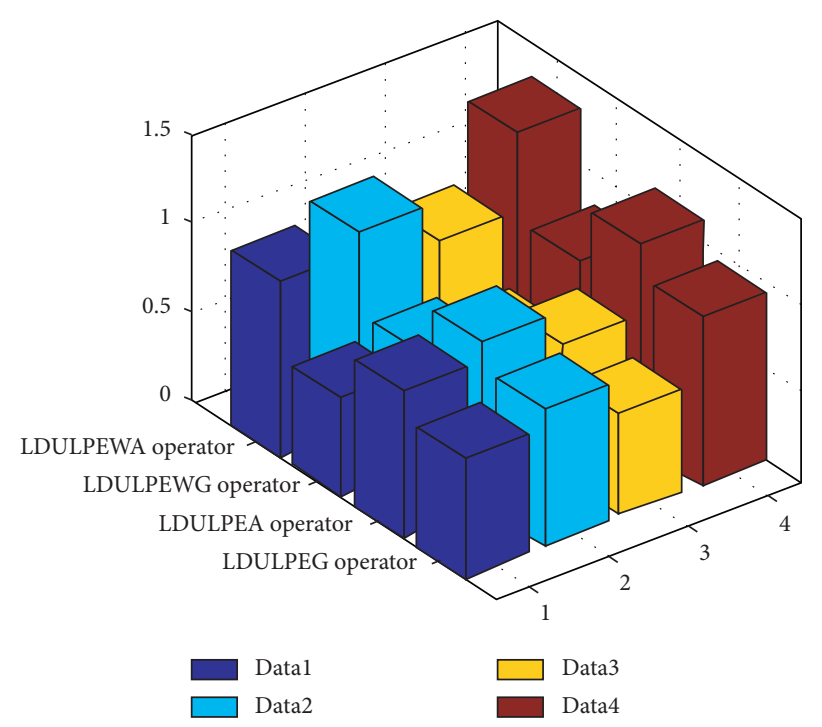

FIgURE 2: Geometrical expressions of the values in Table 3.

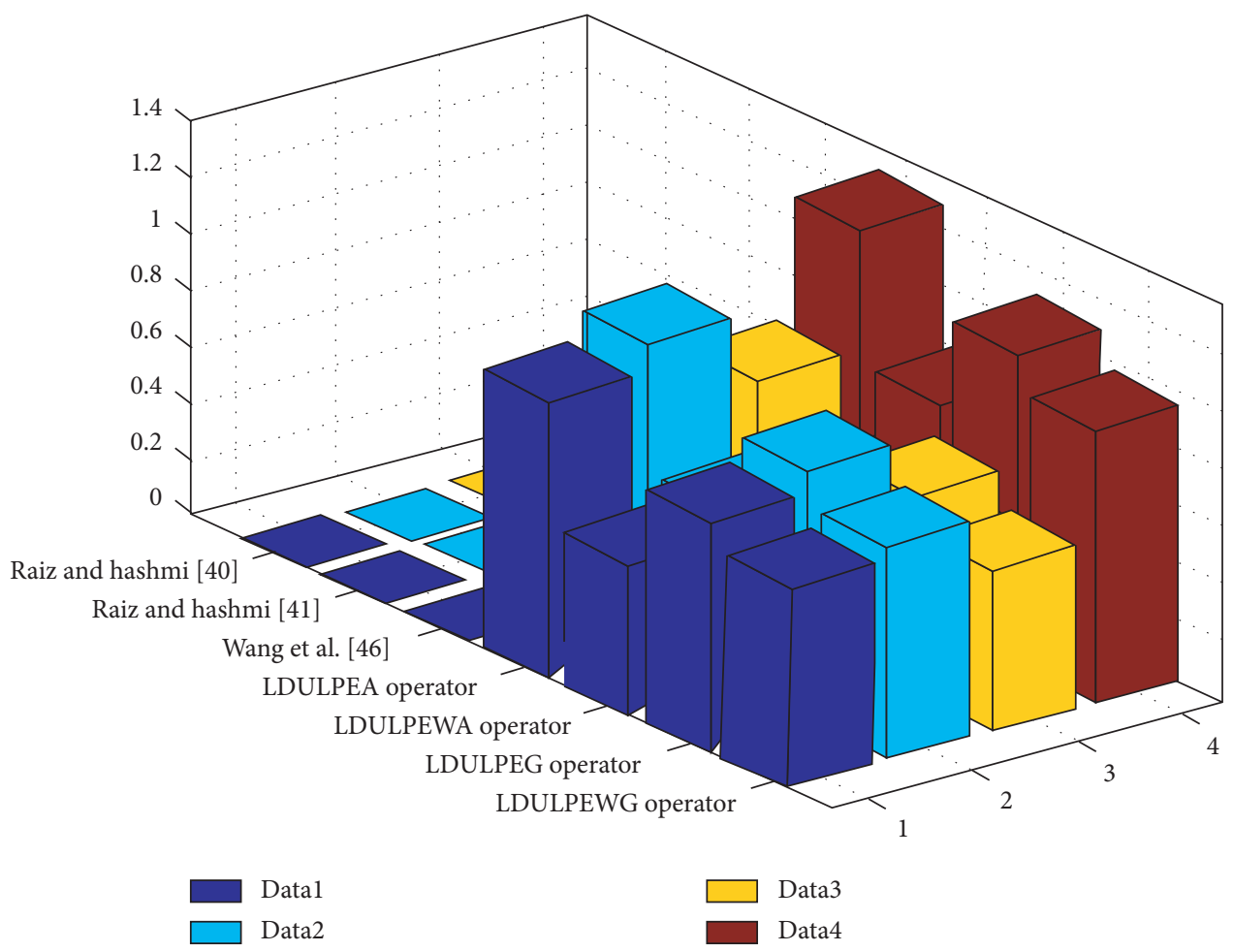

FIgURE 3: Geometrical expressions of the information in Table 5.

The results portrayed in Table 5 are further described geometrically in Figure 3.

As shown above, we chose the linear Diophantine uncertain linguistic types of information, so the operators investigated by Riaz and Hashmi [39] are not able to resolve it. However, if we decide the exiting types of information, then the investigated sort of information can cope with it. Therefore, the investigated operators based on LDULSs are more powerful to determine the rationality and consistency of the developed operators.

\section{Conclusion}

LDULS is a modified variety of the fuzzy set (FS) to manage problematic and inconsistent information in actual life troubles. LDULS covers the grade of truth, grade of falsity, and their reference parameters with ULT with $0 \leq \alpha_{\mathrm{AMG}}$ $u_{\mathrm{AMG}}(x)+\beta_{\mathrm{ANG}} v_{\mathrm{AMG}}(x) \leq 1$, where $0 \leq \alpha_{\mathrm{AMG}}+\beta_{\mathrm{ANG}} \leq 1$. In this manuscript, the principle of LDULS and their useful laws are elaborated. Additionally, PEAO is a conventional sort of $\mathrm{AO}$ utilized in innovative decision-making troubles, 
which is effective to aggregate the family of numerical elements. To determine the interrelationship between any numbers of arguments, we elaborate on the LDULPEA, LDULPEWA, LDULPEG, and LDULPEWG operators and discussed their useful results. Conclusively, a decisionmaking methodology is utilized for the MADM dilemma with elaborated information. A sensible illustration is specified to demonstrate the accessibility and rewards of the intended technique by comparison with certain prevailing techniques. The intended AOs are additional comprehensive than the prevailing ones to exploit the ambiguous and inaccurate knowledge. Numerous remaining operators are chosen as individual incidents of the suggested one. Ultimately, the supremacy and advantages of the elaborated operators are also discussed with the help of geometrical form to show the validity and consistency of explored operators.

In further research, considering the superiority of new LDULSs, we can extend them to some other spherical fuzzy sets [43], spherical linear Diophantine fuzzy sets [44], complex spherical fuzzy sets [45], aggregation operators [42], such as power mean aggregation operators, Bonferroni mean operators, Heronian mean operators, different methods [46-50], and so on [51].

\section{Data Availability}

No data were used to support this study.

\section{Conflicts of Interest}

The authors declare that they have no conflicts of interest.

\section{Acknowledgments}

The authors are grateful to the Deanship of Scientific Research, King Saud University, for funding through Vice Deanship of Scientific Research Chairs.

\section{References}

[1] L. A. Zadeh, "Fuzzy sets," Information and Control, vol. 8, no. 3, pp. 338-353, 1965.

[2] T. Mahmood, "A novel approach towards bipolar soft sets and their applications," Journal of mathematics, vol. 202011 pages, Article ID 4690808, 2020.

[3] J. Somjanta, N. Thuekaew, P. Kumpeangkeaw, and A. Iampan, "Fuzzy sets in UP-algebras," Annals of Fuzzy Mathematics and Informatics, vol. 12, no. 6, pp. 739-756, 2016.

[4] N. Noppharat Dokkhamdang, A. Aiyared Iampan, and A. Akekarin Kesorn, "Generalized fuzzy sets in UP-algebras," Annals of Fuzzy Mathematics and Informatics, vol. 16, no. 2, pp. 171-190, 2018.

[5] K. Tanamoon, S. Sripaeng, and A. Iampan, "Q-fuzzy sets in UP-algebras," Songklanakarin Journal of Science and Technology, vol. 40, no. 1, pp. 9-29, 2018.

[6] K. Kawila, C. Udomsetchai, and A. Iampan, "Bipolar fuzzy UP-algebras," Mathematical and Computational Applications, vol. 23, no. 4, p. 69, 2018.
[7] T. Mahmood and K. Hayat, "Characterizations of hemi-rings by their bipolar-valued fuzzy h-ideals," Information Sciences Letters, vol. 4, no. 2, p. 51, 2015.

[8] T. Mahmood, Z. U. Rehman, and A. Sezgin, "Lattice ordered soft near rings," The Korean Journal of Mathematics, vol. 26, no. 3, pp. 503-517, 2018.

[9] K. T. Atanassov, "Intuitionistic fuzzy sets," Fuzzy Sets and Systems, vol. 20, no. 1, pp. 87-96, 1986.

[10] I. Beg and T. Rashid, "Group decision making using intuitionistic hesitant fuzzy sets," International Journal of Fuzzy Logic and Intelligent Systems, vol. 14, no. 3, 2014.

[11] K. T. Atanassov, "Interval valued intuitionistic fuzzy sets," in Intuitionistic Fuzzy SetsPhysica, Heidelberg, Germany, 1999.

[12] R. Kumari and A. R. Mishra, "Multi-criteria COPRAS method based on parametric measures for intuitionistic fuzzy sets: application of green supplier selection," Iranian Journal of Science and Technology, Transactions of Electrical Engineering, vol. 44, pp. 1-18, 2020.

[13] C. Jana and M. Pal, "Application of bipolar intuitionistic fuzzy soft sets in decision making problem," International Journal of Fuzzy System Applications, vol. 7, no. 3, pp. 32-55, 2018.

[14] B. P. Joshi and S. Kumar, "Fuzzy time series model based on intuitionistic fuzzy sets for empirical research in stock market," International Journal of Applied Evolutionary Computation, vol. 3, no. 4, pp. 71-84, 2012.

[15] S. Fu, X.-l. Qu, H.-j. Zhou, and G.-b. Fan, "A multi-attribute decision-making model using interval-valued intuitionistic fuzzy numbers and attribute correlation," International Journal of Enterprise Information Systems, vol. 14, no. 1, pp. 21-34, 2018.

[16] S. Meng and Y. He, "Generalized scaled prioritized intuitionistic fuzzy geometric interaction aggregation operators and their applications to the selection of cold chain logistics enterprises," International Journal of Fuzzy System Applications, vol. 7, no. 1, pp. 1-21, 2018.

[17] R. R. Yager, "Pythagorean fuzzy subsets," in Proceedings of the 2013 joint IFSA world congress and NAFIPS annual meeting (IFSA/NAFIPS), pp. 57-61, IEEE, Edmonton, Canada, June 2013.

[18] H. Garg, "Linguistic Pythagorean fuzzy sets and its applications in multiattribute decision-making process," International Journal of Intelligent Systems, vol. 33, no. 6, pp. 1234-1263, 2018.

[19] G. Wei and Y. Wei, "Similarity measures of Pythagorean fuzzy sets based on the cosine function and their applications," International Journal of Intelligent Systems, vol. 33, no. 3, pp. 634-652, 2018.

[20] F. Xiao and W. Ding, "Divergence measure of Pythagorean fuzzy sets and its application in medical diagnosis," Applied Soft Computing, vol. 79, pp. 254-267, 2019.

[21] K. Ullah, T. Mahmood, Z. Ali, and N. Jan, "On some distance measures of complex Pythagorean fuzzy sets and their applications in pattern recognition," Complex \& Intelligent Systems, vol. 6, no. 1, pp. 15-27, 2020.

[22] Z. Li and M. Lu, "Some novel similarity and distance measures of pythagorean fuzzy sets and their applications," Journal of Intelligent \& Fuzzy Systems, vol. 37, no. 2, pp. 1781-1799, 2019.

[23] H. Garg, "A novel improved accuracy function for interval valued Pythagorean fuzzy sets and its applications in the decision-making process," International Journal of Intelligent Systems, vol. 32, no. 12, pp. 1247-1260, 2017.

[24] M. S. Yang and Z. Hussain, "Fuzzy entropy for pythagorean fuzzy sets with application to multicriterion decision making," Complexity, vol. 2018, Article ID 2832839, 14 pages, 2018. 
[25] R. R. Yager, "Generalized orthopair fuzzy sets," IEEE Transactions on Fuzzy Systems, vol. 25, no. 5, pp. 1222-1230, 2016.

[26] M. I. Ali, "Another view on q-rung orthopair fuzzy sets," International Journal of Intelligent Systems, vol. 33, no. 11, pp. 2139-2153, 2018.

[27] P. Liu and P. Wang, "Some q-rung orthopair fuzzy aggregation operators and their applications to multiple-attribute decision making," International Journal of Intelligent Systems, vol. 33, no. 2, pp. 259-280, 2018.

[28] X. Peng and L. Liu, "Information measures for $q$-rung orthopair fuzzy sets," International Journal of Intelligent Systems, vol. 34, no. 8, pp. 1795-1834, 2019.

[29] P. Wang, J. Wang, G. Wei, and C. Wei, "Similarity measures of q-rung orthopair fuzzy sets based on cosine function and their applications," Mathematics, vol. 7, no. 4, p. 340, 2019.

[30] Z. Ali and T. Mahmood, "Maclaurin symmetric mean operators and their applications in the environment of complex q-rung orthopair fuzzy sets," Computational and Applied Mathematics, vol. 39, pp. 1-27, 2020.

[31] D. Liu, X. Chen, and D. Peng, "Some cosine similarity measures and distance measures between $\mathrm{q}$-rung orthopair fuzzy sets," International Journal of Intelligent Systems, vol. 34, no. 7, pp. 1572-1587, 2019.

[32] P. Liu and P. Wang, "Multiple-attribute decision-making based on Archimedean Bonferroni operators of q-rung orthopair fuzzy numbers," IEEE Transactions on Fuzzy Systems, vol. 27, no. 5, pp. 834-848, 2018.

[33] M. Lin, X. Li, and L. Chen, "Linguisticq-rung orthopair fuzzy sets and their interactional partitioned Heronian mean aggregation operators," International Journal of Intelligent Systems, vol. 35, no. 2, pp. 217-249, 2020.

[34] H. Garg, "A new possibility degree measure for intervalvalued q-rung orthopair fuzzy sets in decision-making," International Journal of Intelligent Systems, vol. 36, no. 1, pp. 526-557, 2021.

[35] H. Garg, J. Gwak, T. Mahmood, and Z. Ali, "Power aggregation operators and VIKOR methods for complex q-rung orthopair fuzzy sets and their applications," Mathematics, vol. 8, no. 4, p. 538, 2020.

[36] L. A. Zadeh, "The concept of a linguistic variable and its application to approximate reasoning-I," Information Sciences, vol. 8, no. 3, pp. 199-249, 1975.

[37] F. Herrera and L. Martínez, “A 2-tuple fuzzy linguistic representation model for computing with words," IEEE Transactions on Fuzzy Systems, vol. 8, no. 6, pp. 746-752, 2000.

[38] P. Liu and F. Jin, "Methods for aggregating intuitionistic uncertain linguistic variables and their application to group decision making," Information Sciences, vol. 205, pp. 58-71, 2012.

[39] M. Riaz and M. R. Hashmi, "Linear diophantine fuzzy set and its applications towards multi-attribute decision-making problems," Journal of Intelligent \& Fuzzy Systems, vol. 37, no. 4, pp. 5417-5439, 2019.

[40] M. Riaz, M. R. Hashmi, H. Kalsoom, D. Pamucar, and Y.-M. Chu, "Linear diophantine fuzzy soft rough sets for the selection of sustainable material handling equipment," Symmetry, vol. 12, no. 8, p. 1215, 2020.

[41] H. Kamaci, "Linear diophantine fuzzy algebraic structures," Journal of Ambient Intelligence and Humanized Computing, pp. 1-21, 2021.

[42] L. Wang, H. Garg, and N. Li, "Pythagorean fuzzy interactive Hamacher power aggregation operators for assessment of express service quality with entropy weight," Soft Computing, vol. 25, no. 2, pp. 973-993, 2021.

[43] T. Mahmood, K. Ullah, Q. Khan, and N. Jan, "An approach toward decision-making and medical diagnosis problems using the concept of spherical fuzzy sets," Neural Computing and Applications, vol. 31, no. 11, pp. 7041-7053, 2019.

[44] M. Riaz, M. Raza Hashmi, D. Pamucar, and Y. Chu, "Spherical linear Diophantine fuzzy sets with modeling uncertainties in MCDM," Computer Modeling in Engineering \& Sciences, vol. 126, no. 3, pp. 1125-1164, 2021.

[45] Z. Ali, T. Mahmood, and M.-S. Yang, "TOPSIS method based on complex spherical fuzzy sets with Bonferroni mean operators," Mathematics, vol. 8, no. 10, p. 1739, 2020.

[46] G. Wei, Y. He, F. Lei, J. Wu, C. Wei, and Y. Guo, "Green supplier selection with an uncertain probabilistic linguistic MABAC method," Journal of Intelligent \& Fuzzy Systems, vol. 39, no. 3, pp. 3125-3136, 2020.

[47] G. Wei, J. Wang, J. Lu et al., "VIKOR method for multiple criteria group decision making under 2-tuple linguistic neutrosophic environment," Economic Research-Ekonomska Istraživanja, vol. 33, no. 1, pp. 3185-3208, 2020.

[48] L. Xiao, S. Zhang, G. Wei et al., "Green supplier selection in steel industry with intuitionistic fuzzy Taxonomy method," Journal of Intelligent \& Fuzzy Systems, vol. 39, no. 5, pp. 7247-7258, 2020.

[49] G. Wei, C. Wei, and Y. Guo, "EDAS method for probabilistic linguistic multiple attribute group decision making and their application to green supplier selection," Soft Computing, pp. 1-9, 2021.

[50] G. Wei, J. Wu, Y. Guo, J. Wang, and C. Wei, “An extended COPRAS model for multiple attribute group decision making based on single-valued neutrosophic 2-tuple linguistic environment," Technological and Economic Development of Economy, vol. 27, no. 2, pp. 353-368, 2021.

[51] D. Molodtsov, "Soft set theory-first results," Computers \& Mathematics with Applications, vol. 37, no. 4-5, pp. 19-31, 1999. 